\title{
A high performance fifth-order multistep WENO scheme
}

\author{
Fangjun Zeng1,2(D) | Yiqing Shen ${ }^{1,2} \mid$ Shengping Liu',2(D) $\mid$ Li Liu $^{3}$
}

\author{
${ }^{1}$ State Key Laboratory of High \\ Temperature Gas Dynamics, Institute of \\ Mechanics, Chinese Academy of Sciences, \\ Beijing, China \\ ${ }^{2}$ School of Engineering Science, \\ University of Chinese Academy of \\ Sciences, Beijing, China \\ ${ }^{3}$ Institute of Applied Physics and \\ Computational Mathematics, Beijing, \\ China
}

\section{Correspondence}

Yiqing Shen, State Key Laboratory of High Temperature Gas Dynamics, Institute of Mechanics, Chinese Academy of Sciences, No.15 Beisihuanxi Road, Beijing 100190,

China.

Email:yqshen@imech.ac.cn

\section{Funding information}

NSAF, Grant/Award Number: U1530145; NKRDPC, Grant/Award Number: 2016YFA0401200; SCP, Grant/Award Number: TZ2016002; NSFC, Grant/Award Number: 11872067 and 91852203

\begin{abstract}
Summary
Many efforts have been made to improve the accuracy of the conventional weighted essentially nonoscillatory (WENO) scheme at transition points (connecting a smooth region and a discontinuity point). This paper analyzes these works and further develops a more effective multistep WENO scheme. Theoretical analysis and numerical results show that the new scheme not only improves the accuracy by one order higher than the traditional fifth-order WENO schemes at transition point but also maintains the fifth-order accuracy in smooth regions even at critical point where the first derivative vanishes.
\end{abstract}

\section{KEYWORDS}

critical point, high performance, low-dissipation, multistep WENO scheme, transition point

\section{1 | INTRODUCTION}

The weighted essentially nonoscillatory (WENO) schemes have been extensively studied and applied in computational fluid dynamics since it was first proposed by Liu et al. ${ }^{1}$ The distinctive advantage of the WENO scheme is that it not only can achieve uniform high-order accuracy in the smooth areas but can also robustly capture shock waves in the discontinuous regions. Jiang and $\mathrm{Shu}^{2}$ proposed a classical smoothness indicator to calculate the nonlinear weights, with which the WENO (WENO-JS) scheme constructed from the $r$ th order essentially nonoscillatory (ENO) scheme can obtain $(2 r-1)$ th order accuracy. A class of WENO schemes higher than the fifth order is designed by Balsara and Shu ${ }^{3}$ and by Gerolymos et $\mathrm{al}^{4}$

Henrick et $\mathrm{l}^{5}$ derived the necessary and sufficient conditions (NSC) for fifth-order convergence of a fifth-order WENO scheme and pointed out that the fifth-order WENO-JS scheme was reduced to the third-order accuracy at critical points where the first derivative vanishes, and then, the WENO-M scheme was constructed by modifying the weights of WENO-JS with a mapping function. Borges et $\mathrm{al}^{6}$ proposed the WENO-Z scheme by constructing a high-order smoothness indicator to compute the nonlinear weights. Castro et $\mathrm{al}^{7}$ constructed higher order WENO-Z schemes. Ha et $\mathrm{al}^{8}$ and Fan et $\mathrm{al}^{9}$ further improved the accuracy of WENO-Z by constructing higher order global smoothness indicators and developed the WENO-NS and WENO-Z $\eta$ schemes, respectively. On the other hand, Levy et al ${ }^{10-12}$ presented a series of central WENO schemes, in which a two-dimensional (2D) high-order interpolant is reconstructed from cell averages by taking a 
convex combination of building blocks. Martin et $\mathrm{a}^{13}$ proposed a symmetric WENO method by means of a new candidate stencil. Balsara et $\mathrm{al}^{14}$ developed a new class of WENO schemes with adaptive order for hyperbolic conservation laws.

Different from improving the accuracy of fifth-order WENO schemes at critical points, Shen and Zha ${ }^{15}$ first pointed out that the accuracy of the traditional fifth-order WENO schemes is of only second order at transition point (connecting a smooth region and a discontinuity point) and then introduced two fourth-order fluxes combined with an estimation of smoothness/nonsmoothness of two adjacent four-point stencils to improve the accuracy near discontinuities. Subsequently, Shen et $\mathrm{al}^{16}$ developed a multistep WENO scheme, which can improve the accuracy of the fifth-order WENO schemes near discontinuities without reducing the accuracy in smooth regions even at critical points. Peng and Shen ${ }^{17}$ extended the multistep strategy to improve the accuracy of the compact reconstructed WENO (CRWENO) scheme ${ }^{18}$ at transition points. However, in the multistep WENO scheme, six weights are calculated and the mapping function proposed by Henrick et $\mathrm{al}^{5}$ is needed, which make the algorithm inefficient. In order to enhance the computational efficiency of the multistep WENO scheme, Ma et al ${ }^{19}$ proposed an improved multistep WENO scheme and its extension to higher orders of accuracy. Compared with the multistep WENO scheme, although the improved scheme can greatly improve the computational efficiency, the numerical results show that it cannot effectively reduce the dissipation near the discontinuities and its accuracy at critical point is of fourth order. Recently, van Lith et $\mathrm{al}^{20}$ developed a class of embedded WENO scheme. The scheme is simple and provides a choice to improve the accuracy of the WENO-Z scheme at a transition point. In fact, the corresponding embedded WENO-Z scheme is equivalent to multiplying local smoothness indicators by different constants. However, this way, may make the unbalanced contribution of the three substencils of fifth-order WENO scheme and hence results in accuracy decreased in smooth regions. How to develop high performance multistep WENO schemes is still an open issue.

In this paper, based on the analysis of the existing WENO schemes, we further develop the multistep weighting method. Analysis shows that the new method improves the accuracy at transition points, and the calculated weights satisfy the NSCs for the fifth-order convergence in smooth regions even at critical points. Meanwhile, similar to the improved multistep WENO scheme proposed by Ma et al, ${ }^{19}$ the present method only needs to calculate three nonlinear weights and does not require a mapping function, which makes the algorithm more efficient than the original multistep WENO scheme.

The rest of this paper is organized as follows: several conventional fifth-order WENO schemes and accuracy analysis of transition point are briefly introduced in Section 2 . The new scheme is proposed and a detailed analysis about its accuracy at both critical and transition points is given in Section 3. In Section 4, several numerical examples are presented to demonstrate the efficiency, robustness, and low dissipation properties of the proposed scheme. Some conclusions of this paper are given in Section 5.

\section{2 | FIFTH-ORDER WENO SCHEMES}

In this section, the one-dimensional hyperbolic conservation laws is used as a model equation to describe the numerical method

$$
\frac{\partial u}{\partial t}+\frac{\partial f(u)}{\partial x}=0
$$

where $u(x, t)$ is the conservative variable, and $f(u)$ is the flux function.

The semidiscretization form of Equation (1) can be written as

$$
\frac{d u_{i}}{d t}=-\frac{\hat{f}_{i+1 / 2}-\hat{f}_{i-1 / 2}}{\Delta x}
$$

where $\Delta x$ is an uniform grid size, $\hat{f}_{i \pm 1 / 2}$ is the numerical flux.

\section{1 | The traditional fifth-order WENO schemes}

The numerical flux of a fifth-order WENO scheme can be written as

$$
\hat{f}_{i+\frac{1}{2}}=\sum_{k=0}^{2} \omega_{k} \hat{f}_{i+\frac{1}{2}}^{k},
$$


where $\hat{f}_{i+1 / 2}^{k}$ is the third-order flux on the substencil $S_{k}=\{i+k-2, i+k-1, i+k\}$, and given by

$$
\left\{\begin{array}{l}
\hat{f}_{i+\frac{1}{2}}^{0}=\frac{1}{3} f_{i-2}-\frac{7}{6} f_{i-1}+\frac{11}{6} f_{i}, \\
\hat{f}_{i+\frac{1}{2}}^{1}=-\frac{1}{6} f_{i-1}+\frac{5}{6} f_{i}+\frac{1}{3} f_{i+1}, \\
\hat{f}_{i+\frac{1}{2}}^{2}=\frac{1}{3} f_{i}+\frac{5}{6} f_{i+1}-\frac{1}{6} f_{i+2},
\end{array}\right.
$$

$\omega_{k}$ is the nonlinear weights. In the work of Jiang and Shu, ${ }^{2}$ the weight function is defined as

$$
\omega_{k}=\frac{\alpha_{k}}{\alpha_{0}+\alpha_{1}+\alpha_{2}}, \alpha_{k}=\frac{c_{k}}{\left(\beta_{k}+\varepsilon\right)^{2}}, k=0,1,2,
$$

where $c_{0}=\frac{1}{10}, c_{1}=\frac{6}{10}, c_{2}=\frac{3}{10}$ are the ideal weights. The parameter $\varepsilon$ is introduced to avoid the division by zero. A detailed analysis in the work of Henrick et $\mathrm{al}^{5}$ shows that the relatively large value for parameter $\varepsilon$ has an influence on the accuracy of the WENO scheme, especially near critical points. Henrick et al $^{5}$ suggested a value of $\varepsilon$ slightly larger than the square root of the smallest positive number allowed for a particular machine. Even so, in order to achieve better order of accuracy, in the more recent literatures, ${ }^{21-23}$ there have been a lot of improvements on the choice of $\varepsilon$, for example, the parameter $\varepsilon$ is taken as a function of $\Delta x . \beta_{k}$ is the smoothness indicator on substencil $S_{k}$. Jiang and Shu proposed a classical formula for $\beta_{k}$ as follows:

$$
\beta_{k}=\sum_{l=1}^{r-1} \int_{x_{i-\frac{1}{2}}}^{x_{i+\frac{1}{2}}}(\Delta x)^{2 l-1}\left(\frac{d^{l} \hat{f}^{k}(x)}{d x^{l}}\right)^{2} d x .
$$

The explicit form of $\beta_{k}$ for the fifth-order WENO scheme $(r=3)$ can be expressed as

$$
\begin{aligned}
& \beta_{0}=\frac{13}{12}\left(f_{i-2}-2 f_{i-1}+f_{i}\right)^{2}+\frac{1}{4}\left(f_{i-2}-4 f_{i-1}+3 f_{i}\right)^{2}, \\
& \beta_{1}=\frac{13}{12}\left(f_{i-1}-2 f_{i}+f_{i+1}\right)^{2}+\frac{1}{4}\left(f_{i+1}-f_{i-1}\right)^{2}, \\
& \beta_{2}=\frac{13}{12}\left(f_{i}-2 f_{i+1}+f_{i+2}\right)^{2}+\frac{1}{4}\left(3 f_{i}-4 f_{i+1}+f_{i+2}\right)^{2},
\end{aligned}
$$

and their Taylor series expansions at $x_{i}$ are

$$
\begin{aligned}
& \beta_{0}=f_{i}^{\prime 2} \Delta x^{2}+\left(\frac{13}{12} f_{i}^{\prime \prime 2}-\frac{2}{3} f_{i}^{\prime} f_{i}^{\prime \prime \prime}\right) \Delta x^{4}+\left(-\frac{13}{6} f_{i}^{\prime \prime} f_{i}^{\prime \prime \prime}+\frac{1}{2} f_{i}^{\prime} f_{i}^{(4)}\right) \Delta x^{5}+O\left(\Delta x^{6}\right), \\
& \beta_{1}=f_{i}^{\prime 2} \Delta x^{2}+\left(\frac{13}{12} f_{i}^{\prime \prime 2}+\frac{1}{3} f_{i}^{\prime} f_{i}^{\prime \prime \prime}\right) \Delta x^{4}+O\left(\Delta x^{6}\right), \\
& \beta_{2}=f_{i}^{\prime 2} \Delta x^{2}+\left(\frac{13}{12} f_{i}^{\prime \prime 2}-\frac{2}{3} f_{i}^{\prime} f_{i}^{\prime \prime \prime}\right) \Delta x^{4}+\left(\frac{13}{6} f_{i}^{\prime \prime} f_{i}^{\prime \prime \prime}-\frac{1}{2} f_{i}^{\prime} f_{i}^{(4)}\right) \Delta x^{5}+O\left(\Delta x^{6}\right) .
\end{aligned}
$$

Henrick et $\mathrm{al}^{5}$ provided a detailed analysis about the accuracy of the fifth-order WENO scheme of Jiang and Shu (WENO-JS) and derived the NSCs on the weights for fifth-order convergence as

$$
\begin{aligned}
\sum_{k=0}^{2} A_{k}\left(\omega_{k}^{+}-\omega_{k}^{-}\right) & =O\left(\Delta x^{3}\right), \\
\omega_{k}^{ \pm}-c_{k} & =O\left(\Delta x^{2}\right),
\end{aligned}
$$

and a simple sufficient condition, which is given as

$$
\omega_{k}^{ \pm}-c_{k}=O\left(\Delta x^{3}\right)
$$

where the superscripts \pm correspond to the numerical fluxes $\hat{f}_{i \pm 1 / 2}$, respectively. In order to satisfy the aforementioned conditions, a mapping function is proposed in the work of Henrick et $\mathrm{al}^{5}$ as

$$
g_{k}(\omega)=\frac{\omega\left(c_{k}+c_{k}^{2}-3 c_{k} \omega+\omega^{2}\right)}{c_{k}^{2}+\omega\left(1-2 c_{k}\right)},
$$

and an improved WENO (WENO-M) scheme is developed by using $g_{k}(\omega)$.

Borges et $\mathrm{al}^{6}$ proposed a simple way to calculate the weights as

$$
\omega_{k}=\frac{\alpha_{k}}{\alpha_{0}+\alpha_{1}+\alpha_{2}}, \alpha_{k}=c_{k}\left(1+\left(\frac{\tau_{5}}{\beta_{k}+\varepsilon}\right)^{q}\right), k=0,1,2
$$


where $\tau_{5}=\left|\beta_{0}-\beta_{2}\right|$ can be called as a high-order smoothness indicator. In smooth regions, the Taylor expansion of $\tau_{5}$ at $x_{i}$ gives

$$
\tau_{5}= \begin{cases}\left|\frac{13}{3} f_{i}^{\prime \prime} f_{i}^{\prime \prime \prime}-f_{i}^{\prime} f_{i}^{(4)}\right| \Delta x^{5}+O\left(\Delta x^{7}\right), & f_{i}^{\prime} \neq 0, \\ \left|\frac{13}{3} f_{i}^{\prime \prime} f_{i}^{\prime \prime \prime}\right| \Delta x^{5}+O\left(\Delta x^{7}\right), & f_{i}^{\prime}=0 .\end{cases}
$$

As analyzed by Borges et al, ${ }^{6}$ the convergence order of this new WENO (WENO-Z) scheme at critical points is fourth and fifth orders if the power $q$ takes the value of 1 and 2, respectively. However, for solutions containing the discontinuities, increasing $q$ makes the scheme more dissipative.

\section{2 | Accuracy analysis at transition point}

Shen and $\mathrm{Zha}^{15}$ provided a detailed analysis about the accuracy of fifth-order WENO schemes near discontinuities and found that they fail to get the optimal order at transition points (see Figure 1).

For completeness, we briefly analyze the accuracy of the transition point here. Without loss of generality, we assume that a discontinuity is located at $\left[x_{i+1}, x_{i+2}\right]$ (see Figure 1), ie, the rightmost stencil $S_{2}=\left\{x_{i}, x_{i+1}, x_{i+2}\right\}$ of the fifth-order WENO scheme. In this situation, analysis in the work of Shen and Zha ${ }^{15}$ shows that the nonlinear weight $\omega_{2}$ of the WENO-JS or WENO-Z scheme decreases to an essentially zero value, whereas $\omega_{0}$ and $\omega_{1}$ approach $\frac{1}{7}$ and $\frac{6}{7}$, respectively. Therefore, applying Taylor series expansion, Equation (3) can be written as

$$
\hat{f}_{i+\frac{1}{2}}=\frac{1}{7} \hat{f}_{i+\frac{1}{2}}^{0}+\frac{6}{7} \hat{f}_{i+\frac{1}{2}}^{1}=\frac{2 f_{i-2}-13 f_{i-1}+41 f_{i}+12 f_{i+1}}{42}=h_{i+\frac{1}{2}}+O\left(\Delta x^{3}\right) .
$$

However, for calculating the flux $\hat{f}_{i-\frac{1}{2}}$, the stencil $S_{5}=\left\{x_{i-3}, x_{i-2}, \ldots, x_{i+1}\right\}$ is smooth; hence, the fifth-order numerical flux can be obtained as

$$
\hat{f}_{i-\frac{1}{2}}=\frac{2 f_{i-3}-13 f_{i-2}+47 f_{i-1}+27 f_{i}-3 f_{i+1}}{60}=h_{i-\frac{1}{2}}+O\left(\Delta x^{5}\right) .
$$

Subtracting Equation (14) from Equation (15) gives

$$
\frac{\hat{f}_{i+\frac{1}{2}}-\hat{f}_{i-\frac{1}{2}}}{\Delta x}=f_{i}^{\prime}+O\left(\Delta x^{2}\right) .
$$

From Equation (16), we can see that the accuracy of the WENO-JS and WENO-Z schemes at transition point is of only second order. As pointed out in the work of Shen et al, ${ }^{16}$ a fourth-order numerical flux can be constructed by using a

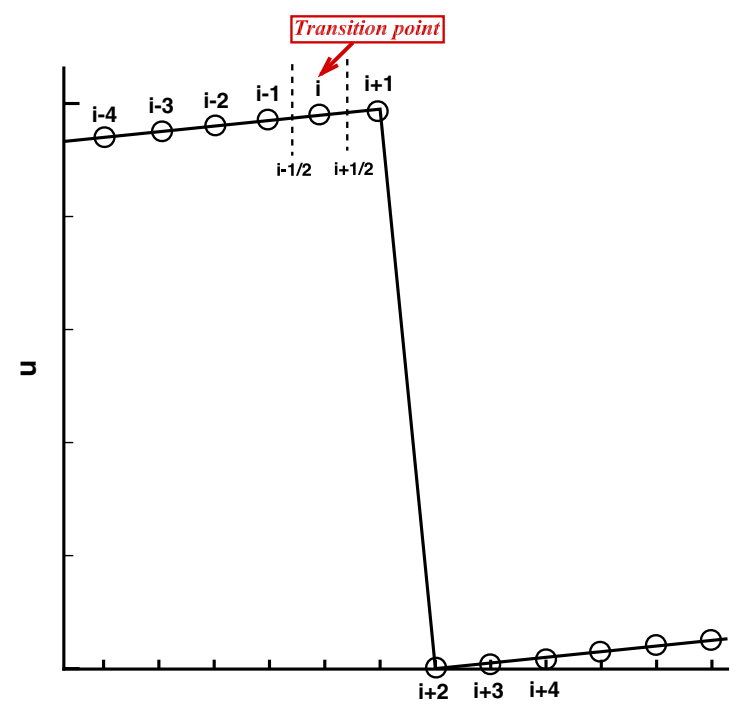

FIGURE 1 The sketch of transition point [Colour figure can be viewed at wileyonlinelibrary.com] 
smooth stencil with four points. That is to say, in the smooth stencil $S_{4}=\left\{x_{i-2}, x_{i-1}, x_{i}, x_{i+1}\right\}$, the numerical flux $\hat{f}_{i+\frac{1}{2}}$ can be rewritten as

$$
\hat{f}_{i+\frac{1}{2}}=c_{0}^{4,0} \hat{f}_{i+\frac{1}{2}}^{0}+c_{1}^{4,0} \hat{f}_{i+\frac{1}{2}}^{1}=\frac{f_{i-2}-5 f_{i-1}+13 f_{i}+3 f_{i+1}}{12}=h_{i+\frac{1}{2}}+O\left(\Delta x^{4}\right),
$$

where $c_{0}^{4,0}=\frac{1}{4}, c_{1}^{4,0}=\frac{3}{4}$ are the optimal weights.

Hence, subtracting Equation (17) from Equation (15) yields

$$
\frac{\hat{f}_{i+\frac{1}{2}}-\hat{f}_{i-\frac{1}{2}}}{\Delta x}=f_{i}^{\prime}+O\left(\Delta x^{3}\right) .
$$

Comparing Equation (16) with Equation (18), we can see that the accuracy at transition point is improved by one order. A symmetric scenario, which the discontinuity is present at $\left[x_{i-2}, x_{i-1}\right]$, ie, the leftmost stencil $S_{0}=\left\{x_{i-2}, x_{i-1}, x_{i}\right\}$ of the fifth-order WENO scheme, can be analyzed similarly as above.

\subsection{Improvements of the accuracy at transition point}

\subsection{1 | Hybrid method}

In the work of Shen and Zha, ${ }^{15}$ a new method is proposed to improve the accuracy at transition point and given as

$$
\hat{f}_{i+\frac{1}{2}}=\left\{\begin{array}{l}
\bar{h}_{0}^{4}, \text { if } \tau_{4}^{0} \leq \min \left(\beta_{0}, \beta_{1}, \beta_{2}\right) \text { and } \tau_{4}^{1}>\min \left(\beta_{0}, \beta_{1}, \beta_{2}\right), \\
\bar{h}_{1}^{4}, \text { if } \tau_{4}^{0}>\min \left(\beta_{0}, \beta_{1}, \beta_{2}\right) \text { and } \tau_{4}^{1} \leq \min \left(\beta_{0}, \beta_{1}, \beta_{2}\right), \\
h^{\text {WENO-Z }, \text { otherwise }}
\end{array}\right.
$$

where

$$
\left\{\begin{array}{l}
\bar{h}_{0}^{4}=\frac{1}{12}\left(f_{i-2}-5 f_{i-1}+13 f_{i}+3 f_{i+1}\right) \\
\bar{h}_{1}^{4}=\frac{1}{12}\left(-f_{i-1}+7 f_{i}+7 f_{i+1}-3 f_{i+2}\right)
\end{array}\right.
$$

are two fourth-order fluxes. $\tau_{4}^{0}$ and $\tau_{4}^{1}$ are defined as $\tau_{4}^{0}=\left|\beta_{0}-\beta_{1}\right|$ and $\tau_{4}^{1}=\left|\beta_{1}-\beta_{2}\right|$, respectively. $h^{\text {WENO-Z }}$ denotes the flux of the WENO-Z scheme. Numerical results in the works of Shen and Zha ${ }^{15}$ demonstrate that the new method is more accurate to resolve the flow solutions near discontinuities.

\subsection{2 | Multistep WENO scheme}

In order to remove the logical statements and construct a better performance scheme, Shen et $\mathrm{a}^{16}{ }^{1}$ developed a multistep WENO scheme which synthetically improved the accuracy of the traditional fifth-order WENO schemes at both critical and transition points. In the first step, two fourth-order fluxes are constructed as

$$
\left\{\begin{array}{l}
h_{0}^{4}=\omega_{0}^{4,0} \hat{f}_{i+\frac{1}{2}}^{0}+\omega_{1}^{4,0} \hat{f}_{i+\frac{1}{2}}^{1} \\
h_{1}^{4}=\omega_{0}^{4,1} \hat{f}_{i+\frac{1}{2}}^{1}+\omega_{1}^{4,1} \hat{f}_{i+\frac{1}{2}}^{2}
\end{array}\right.
$$

where

$$
\omega_{k}^{4, l}=\frac{g_{k}\left(\psi_{k}^{4, l}\right)}{\sum_{k} g_{k}\left(\psi_{k}^{4, l}\right)}, \psi_{k}^{4, l}=\frac{\alpha_{k}^{4, l}}{\sum_{k} \alpha_{k}^{4, l}}, \alpha_{k}^{4, l}=c_{k}^{4, l}\left(1+\frac{\left|\beta_{l+1}-\beta_{l}\right|}{\beta_{l+k}+\varepsilon}\right), \quad k, l=0,1 .
$$

$c_{0}^{4,0}$ and $c_{1}^{4,0}$ are defined in Equation (17); $c_{0}^{4,1}=\frac{1}{2}, c_{1}^{4,1}=\frac{1}{2}$ are the optimal weights; $g_{k}(x)$ is a mapping function in Equation (11). In the second step, the final fifth-order flux is obtained as

$$
\hat{f}_{i+\frac{1}{2}}=\omega_{0} h_{0}^{4}+\omega_{1} h_{1}^{4}
$$


where

$$
\omega_{k}=\frac{g_{k}\left(\psi_{k}\right)}{\sum_{k} g_{k}\left(\psi_{k}\right)}, \quad \psi_{k}=\frac{\alpha_{k}}{\sum_{k} \alpha_{k}}, \quad \alpha_{k}=d_{k}\left(1+\frac{\tau_{5}}{\beta_{k}+\varepsilon}\right), k=0,1
$$

$d_{0}=\frac{2}{5}, d_{1}=\frac{3}{5}$ are the optimal weights; $\tau_{5}$ is the global smoothness indicator of the WENO-Z scheme.

Referring to the work of Shen et $a l,{ }^{16}$ it is easy to see that

$$
\omega_{k}^{4, l}= \begin{cases}c_{k}^{4, l}+O\left(\Delta x^{6}\right), & f_{i}^{\prime} \neq 0, \\ c_{k}^{4, l}+O\left(\Delta x^{3}\right), & f_{i}^{\prime}=0,\end{cases}
$$

and

$$
\omega_{k}= \begin{cases}d_{k}+O\left(\Delta x^{9}\right), & f_{i}^{\prime} \neq 0 \\ d_{k}+O\left(\Delta x^{3}\right), & f_{i}^{\prime}=0\end{cases}
$$

Equation (25) shows that the flux of the multistep WENO scheme can approximate the flux $\bar{h}_{0}^{4}$ and $\bar{h}_{1}^{4}$ in Equation (20) if a discontinuity is located at $\left[x_{i+1}, x_{i+2}\right]$ and $\left[x_{i-2}, x_{i-1}\right]$, respectively. Meanwhile, from Equation (26), we can see that the weights of the multistep WENO scheme satisfy the sufficient condition for the fifth-order convergence in smooth regions even at critical points.

\subsection{3 | Embedded WENO-Z scheme}

Recently, van Lith et $\mathrm{l}^{20}$ proposed a new method to improve the WENO-Z scheme. Their formula for calculating the weights is

$$
\omega_{k}=\frac{\alpha_{k}^{E}}{\alpha_{0}^{E}+\alpha_{1}^{E}+\alpha_{2}^{E}}, \quad \alpha_{k}^{E}=c_{k}\left(1+\mu \tilde{c}_{k}\left(\frac{\tau_{5}}{\beta_{k}+\varepsilon}\right)^{q}\right), \quad k=0,1,2,
$$

where parameters $c_{k}, \beta_{k}, \tau_{5}$, and $\varepsilon$ are the same to the ones of the WENO-Z scheme in Equation (12), $\mu=\frac{1}{4}, q=2 ; \tilde{c}_{1}=1$, $\tilde{c}_{0}=\tilde{c}_{2}=2$ is based on the consideration of improving the accuracy at transition point. Equation (27) shows that the embedded WENO-Z (E-WENO) scheme is equivalent to multiplying local smoothness indicators by different constants, ie, $\beta_{k}^{\prime}=\mu_{k} \beta_{k}\left(\mu_{0}=\mu_{2}=\frac{\sqrt{2}}{2}, \mu_{1}=\frac{1}{2}\right)$.

\subsubsection{Improved multistep WENO scheme}

In order to reduce the computational cost of the multistep WENO scheme, Ma et al ${ }^{19}$ proposed an improved fifth-order multistep WENO (IM-WENO) scheme, in which, the unnormalized weights are defined as

$$
\left\{\begin{array}{l}
\alpha_{0}=c_{0}\left(1+\frac{\tau_{5}}{\beta_{0}+\varepsilon}\right), \\
\alpha_{1}=c_{1}\left(1+\frac{\tau_{5}}{\beta_{0}+\beta_{1}+\varepsilon}+\frac{\tau_{5}}{\beta_{1}+\beta_{2}+\varepsilon}\right), \\
\alpha_{2}=c_{2}\left(1+\frac{\tau_{5}}{\beta_{2}+\varepsilon}\right),
\end{array}\right.
$$

where parameters $c_{k}, \beta_{k}, \tau_{5}$, and $\varepsilon$ are the same as the ones of the WENO-Z scheme in Equation (12)).

\section{3 | THE NEW WENO SCHEME}

\subsection{The accuracy of E-WENO and IM-WENO}

For a smooth solution, by using the Taylor series expansion of $\beta_{k}(8)$ and $\tau_{5}(13)$, the unnormalized weights of the WENO-Z scheme in Equation (12) can be written as

$$
\alpha_{k}=c_{k}\left(1+B \Delta x^{r}+O\left(\Delta x^{r+1}\right)\right),
$$

where $B$ is independent of $k$, and $r=3 q$ and $q$ for $f_{i}^{\prime} \neq 0$ and $f_{i}^{\prime}=0$, respectively. 
Hence, for the WENO-Z scheme, there is

$$
\begin{aligned}
\omega_{k} & =\frac{c_{k}\left(1+B \Delta x^{r}+O\left(\Delta x^{r+1}\right)\right)}{c_{0}\left(1+B \Delta x^{r}+O\left(\Delta x^{r+1}\right)\right)+c_{1}\left(1+B \Delta x^{r}+O\left(\Delta x^{r+1}\right)\right)+c_{2}\left(1+B \Delta x^{r}+O\left(\Delta x^{r+1}\right)\right)} \\
& =c_{k}+O\left(\Delta x^{r+1}\right) .
\end{aligned}
$$

After a simple derivation, it can be found that the Taylor series expansion of the unnormalized weights Equation (28) of the IM-WENO scheme is the same as Equation (29) with $q=1$. Hence, the IM-WENO scheme has the same order as the WENO-Z scheme with $q=1$.

For the E-WENO scheme, the unnormalized weights $\alpha_{k}^{E}$ give

$$
\alpha_{k}^{E}=c_{k}\left(1+B_{k} \Delta x^{r}+O\left(\Delta x^{r+1}\right)\right),
$$

with the parameters $\mu_{k}$ given in the work of van Lith et al, ${ }^{20}$ there is

$$
B_{0}=B_{2} \neq B_{1}
$$

hence, we have

$$
\omega_{k}=c_{k}+O\left(\Delta x^{r}\right)
$$

That means, compared with WENO-Z and IM-WENO, the E-WENO scheme decreases one order for the weights approximating to the ideal weights.

For a solution that contains a discontinuity, for example, which is located at $\left[x_{i+1}, x_{i+2}\right]$, we can analyze the properties of E-WENO and IM-WENO at transition point $x_{i}$. The E-WENO scheme is taken as an example and the parameter $\varepsilon$ takes 0 (since the value of $\varepsilon$ should not destroy the ENO property and in order to simplify the analysis).

Since the discontinuity is located at $\left[x_{i+1}, x_{i+2}\right]$, we have

$$
\beta_{2}=O(1), \beta_{k}=\left\{\begin{array}{ll}
f_{i}^{\prime 2} \Delta x^{2}+O\left(\Delta x^{4}\right), & f_{i}^{\prime} \neq 0, \\
\frac{13}{12} f_{i}^{\prime 2} \Delta x^{4}+O\left(\Delta x^{5}\right), & f_{i}^{\prime}=0,
\end{array} k=0,1, \text { and } \tau_{5}=O(1),\right.
$$

that means

$$
\beta_{2}>\beta_{0}, \beta_{1},
$$

and hence, $\omega_{2} \rightarrow 0$.

Next, we analyze the weights $\omega_{0}$. By using Equation (27), $\omega_{0}$ is calculated as

$$
\omega_{0}=\frac{c_{0}\left(1+\mu \tilde{c}_{0}\left(\frac{\tau_{5}}{\beta_{0}}\right)^{q}\right)}{1+\mu c_{0} \widetilde{c}_{0}\left(\frac{\tau_{5}}{\beta_{0}}\right)^{q}+\mu c_{1}\left(\frac{\tau_{5}}{\beta_{1}}\right)^{q}+\mu c_{2} \widetilde{c}_{2}\left(\frac{\tau_{5}}{\beta_{2}}\right)^{q}} .
$$

Multiplying the numerator and denominator of the right-hand term of Equation (36) by $1 /\left(\mu \tilde{c}_{0}\left(\frac{\tau_{5}}{\beta_{0}}\right)^{q}\right)$, it can be simplified as

$$
\omega_{0}=\frac{c_{0}\left(1+\frac{1}{\mu \tilde{c}_{0}}\left(\frac{\beta_{0}}{\tau_{5}}\right)^{q}\right)}{c_{0}+\frac{c_{1}}{\widetilde{c}_{0}}\left(\frac{\beta_{0}}{\beta_{1}}\right)^{q}+\frac{1}{\mu \tilde{c}_{0}}\left(\frac{\beta_{0}}{\tau_{5}}\right)^{q}+\frac{c_{2} \widetilde{c}_{2}}{\widetilde{c}_{0}}\left(\frac{\beta_{0}}{\beta_{2}}\right)^{q}} .
$$

Substituting Equation (34) and $\tilde{c}_{0}=2$ into Equation (37), we obtain

$$
\omega_{0}=\frac{c_{0}+O\left(\Delta x^{r_{1}}\right)}{c_{0}+\frac{1}{2} c_{1}\left(1+O\left(\Delta x^{r_{2}}\right)\right)+O\left(\Delta x^{r_{1}}\right)},
$$

where, for analyzing convenience, we directly express $\beta_{0} / \beta_{1}$ as $1+O\left(\Delta x^{r_{2}}\right)$ in Equation (38).

Since $c_{0}=1 / 10, c_{1}=6 / 10$, we have

$$
\omega_{0}=c_{0}^{4,0}+O\left(\Delta x^{r}\right)
$$


where $c_{0}^{4,0}=1 / 4$ and $r=\min \left(r_{1}, r_{2}\right)$. According to Equation (34), it is easy to find that

$$
r_{1}= \begin{cases}2 q, & f_{i}^{\prime} \neq 0, \\ 4 q, & f_{i}^{\prime}=0 .\end{cases}
$$

Clearly, $q=2$ can improve the accuracy of the numerator. The key problem is how to improve the accuracy of the denominator. Since $\beta_{2}=O(1)$ and $\tau_{5}=O(1)$, it is clear that $r_{2}$ is decided by $\beta_{0} / \beta_{1}$. From Equation (8), for a smooth solution, there is

$$
\frac{\beta_{k}}{\beta_{j}}=\left\{\begin{array}{ll}
1+O\left(\Delta x^{2}\right), & f_{i}^{\prime} \neq 0, \\
1+O(\Delta x), & f_{i}^{\prime}=0,
\end{array} k \neq j .\right.
$$

A similar formula holds for the two smooth stencils at transition point. Hence, one has that

$$
r_{2}= \begin{cases}2, & f_{i}^{\prime} \neq 0 \\ 1, & f_{i}^{\prime}=0 .\end{cases}
$$

Similarly, we can analyze $\omega_{1}$. Hence, we get

$$
\omega_{k}=\left\{\begin{array}{ll}
c_{k}^{4,0}+O\left(\Delta x^{2}\right), & f_{i}^{\prime} \neq 0, \\
c_{k}^{4,0}+O(\Delta x), & f_{i}^{\prime}=0,
\end{array} \quad k=0,1 .\right.
$$

The IM-WENO scheme can be analyzed similarly, and the same conclusion Equation (43) can be drawn. Please notice that the formula equation (43) is independent of the power $q$.

Equation (43) indicates that both the E-WENO and IM-WENO schemes can theoretically approximate the fourth-order flux $\overrightarrow{h_{0}}$ in Equation (20) when the discontinuity is located at $\left[x_{i+1}, x_{i+2}\right]$. However, numerical results in the works of Ma et $\mathrm{a}^{19}$ and van Lith et $\mathrm{al}^{20}$ and in this paper show that the E-WENO and IM-WENO schemes cannot effectively reduce the dissipation of WENO-Z near the discontinuities. This paper will further develop multistep WENO scheme, which can obtain high-order accuracy in smooth regions and can also decrease the dissipation near the discontinuities.

\section{2 | The new weighting method}

As indicated above, theoretically, the idea of multistep weighting is helpful for improving the accuracy at transition point. However, limited by the condition of $\left(\beta_{k} / \beta_{j}, k \neq j\right)$, the simple linear combination of different $\beta$, such as the formula of Ma et $\mathrm{al}^{19}$ and van Lith et $\mathrm{al},{ }^{20}$ cannot achieve the expected effects. Hence, we propose a new method by using a function $h\left(\beta_{l}, \beta_{1}\right)(l=0,2)$ to replace $\beta_{l}+\beta_{1}$ in Equation (28), and the function should satisfy

$$
h\left(\beta_{l}, \beta_{1}\right)= \begin{cases}2 \beta_{l}+O\left(\Delta x^{\tilde{N}}\right), & \text { if } S_{4}^{l} \text { is a smooth stencil, } \\ \sim \beta_{1}, & \beta_{l} \ll \beta_{1},\end{cases}
$$

where $S_{4}^{0}=\left\{x_{i-2}, \ldots, x_{i+1}\right\}, S_{4}^{2}=\left\{x_{i-1}, \ldots, x_{i+2}\right\}$, and larger $\widetilde{N}$ gives better accuracy for solutions.

For this purpose, a function $h\left(\beta_{l}, \beta_{1}\right)$ is suggested as

$$
h\left(\beta_{l}, \beta_{1}\right)=2 \beta_{l}+\left(\frac{\beta_{l}-\beta_{1}}{\beta_{l}+\beta_{1}+\varepsilon}\right)^{2} \beta_{1}, \quad l=0,2 .
$$

Obviously, the new function equation (45) satisfies the requirements in Equation (44), and $\widetilde{N}=6$ for both the cases of $f_{i}^{\prime}=0$ and $f_{i}^{\prime} \neq 0$. Then, using Equation (45), a new method for calculating the weights is proposed as

$$
\left\{\begin{array}{l}
\alpha_{0}=c_{0}\left(1+\frac{\tau_{5}}{\beta_{0}+\varepsilon}\right), \\
\alpha_{1}=c_{1}\left(1+\frac{\tau_{5}}{h\left(\beta_{0}, \beta_{1}\right)+\varepsilon}+\frac{\tau_{5}}{h\left(\beta_{2}, \beta_{1}\right)+\varepsilon}\right), \\
\alpha_{2}=c_{2}\left(1+\frac{\tau_{5}}{\beta_{2}+\varepsilon}\right),
\end{array}\right.
$$

where parameters $c_{k}, \beta_{k}, \tau_{5}$, and $\varepsilon$ are the same as the ones of the WENO-Z scheme in Equation (12). 
Similarly, for the case of the power parameter $q=2$, the unnormalized weights can be defined as

$$
\left\{\begin{array}{l}
\alpha_{0}=c_{0}\left(1+\frac{\tau_{5}^{2}}{\beta_{0}^{2}+\varepsilon}\right), \\
\alpha_{1}=c_{1}\left(1+\frac{\tau_{5}^{2}}{h\left(\beta_{0}^{2}, \beta_{1}^{2}\right)+\varepsilon}+\frac{\tau_{5}^{2}}{h\left(\beta_{2}^{2}, \beta_{1}^{2}\right)+\varepsilon}\right), \\
\alpha_{2}=c_{2}\left(1+\frac{\tau_{5}^{2}}{\beta_{2}^{2}+\varepsilon}\right) .
\end{array}\right.
$$

Alternatively, one can even use a simpler form

$$
h\left(\beta_{l}^{2}, \beta_{1}^{2}\right)=2 \beta_{l}^{2}+\left(\beta_{l}-\beta_{1}\right)^{2}, l=0,2 .
$$

\subsection{Accuracy analysis of the new scheme}

Assuming the solution is sufficiently smooth in all candidate stencils, and substituting Equations (8) and (13) into Equation (46), we have

$$
\omega_{k}= \begin{cases}c_{k}+O\left(\Delta x^{5}\right), & f_{i}^{\prime} \neq 0, \\ c_{k}+O\left(\Delta x^{2}\right), & f_{i}^{\prime}=0 .\end{cases}
$$

Equation (49) shows that the new weights satisfy the second term of the NSC Equation (9b) for fifth-order convergence in smooth regions even at critical points. Similar to the analysis in the work of Borges et $a l,{ }^{6}$ the first term in the NSC Equation (9a) can be written as

$$
\sum_{k=0}^{2} A_{k}\left(\omega_{k}^{+}-\omega_{k}^{-}\right)=\frac{N^{+} D^{-}-N^{-} D^{+}}{D^{+} D^{-}}=O\left(\Delta x^{S}\right),
$$

where

$$
N^{ \pm}=\tau_{5}^{ \pm} \prod_{k=0}^{2} \bar{\beta}_{k}^{ \pm} \sum_{k=0}^{2} \frac{A_{k}^{ \pm} c_{k}}{\bar{\beta}_{k}^{ \pm}}, \quad D^{ \pm}=\prod_{k=0}^{2} \bar{\beta}_{k}^{ \pm}+\tau_{5}^{ \pm} \prod_{k=0}^{2} \bar{\beta}_{k}^{ \pm} \sum_{k=0}^{2} \frac{c_{k}}{\bar{\beta}_{k}^{ \pm}}
$$

$\bar{\beta}_{k}$ denotes the modified smoothness indicators of the IM-WENO and present schemes. In the IM-WENO scheme,

$$
\bar{\beta}_{0}=\beta_{0}, \bar{\beta}_{1}=\frac{\left(\beta_{0}+\beta_{1}\right)\left(\beta_{1}+\beta_{2}\right)}{\left(\beta_{0}+\beta_{1}\right)+\left(\beta_{1}+\beta_{2}\right)}, \bar{\beta}_{2}=\beta_{2} .
$$

In the present scheme,

$$
\bar{\beta}_{0}=\beta_{0}, \bar{\beta}_{1}=\frac{h\left(\beta_{0}, \beta_{1}\right) h\left(\beta_{2}, \beta_{1}\right)}{h\left(\beta_{0}, \beta_{1}\right)+h\left(\beta_{2}, \beta_{1}\right)}, \bar{\beta}_{2}=\beta_{2},
$$

where the function $h$ is defined in Equation (45).

Thus, with the aid of a symbolic algebra program by substituting the Taylor series expansion of the smoothness indicators $\beta_{k}$ Equation (8), Table 1 gives the value of $s$ in Equation (50). In addition, the process for calculating these values is given in the Appendix.

From Table 1, the first term of the NSC of the IM-WENO scheme is given by

$$
\sum_{k=0}^{2} A_{k}\left(\omega_{k}^{+}-\omega_{k}^{-}\right)= \begin{cases}O\left(\Delta x^{6}\right), & f_{i}^{\prime} \neq 0, \\ O\left(\Delta x^{2}\right), & f_{i}^{\prime}=0 .\end{cases}
$$

TABLE 1 The value of $s$ in Equation (50)

\begin{tabular}{ccccc} 
& \multicolumn{2}{c}{ IM-WENO } & \multicolumn{2}{c}{ Present } \\
& $\boldsymbol{f}_{\boldsymbol{i}}^{\prime} \neq \mathbf{0}$ & $\boldsymbol{f}_{\boldsymbol{i}}^{\prime}=\mathbf{0}$ & $\boldsymbol{f}_{\boldsymbol{i}}^{\prime} \neq \mathbf{0}$ & $\boldsymbol{f}_{\boldsymbol{i}}^{\prime}=\mathbf{0}$ \\
$N^{+} D^{-}-N^{-} D^{+}$ & $O\left(\Delta x^{18}\right)$ & $O\left(\Delta x^{26}\right)$ & $O\left(\Delta x^{20}\right)$ & $O\left(\Delta x^{27}\right)$ \\
$D^{+} D^{-}$ & $O\left(\Delta x^{12}\right)$ & $O\left(\Delta x^{24}\right)$ & $O\left(\Delta x^{12}\right)$ & $O\left(\Delta x^{24}\right)$ \\
$\mathrm{s}$ & 6 & 2 & 8 & 3 \\
\hline
\end{tabular}


However, for the present scheme, there is

$$
\sum_{k=0}^{2} A_{k}\left(\omega_{k}^{+}-\omega_{k}^{-}\right)= \begin{cases}O\left(\Delta x^{8}\right), & f_{i}^{\prime} \neq 0, \\ O\left(\Delta x^{3}\right), & f_{i}^{\prime}=0 .\end{cases}
$$

Equations (49) and (55) indicate that the weights of the present scheme satisfy the NSC for fifth-order convergence in smooth regions including critical points where the first derivative vanishes but the high-order derivative does not, while the weights of the IM-WENO scheme do not satisfy the NSC at critical points, as shown in Equations (30) and (54).

It should be pointed out that, in this paper, only the weighting method with a fixed value for $\varepsilon$ (for example, $\varepsilon=10^{-40}$ ) is applied, so the fifth-order accuracy can be achieved only for the case with the first critical point where $f^{\prime}=0$ and $f^{\prime \prime} \neq 0$. For the higher order critical point, similar as the WENO-Z scheme, ${ }^{6,9}$ the new scheme has only second order. In order to obtain high-order convergence rate, one feasible way is to set $\varepsilon$ proportionally to $\Delta x$ to some power ${ }^{22}$ or design higher order global smoothness indicator. ${ }^{9}$

If the solution is discontinuous on the global stencil, without loss of generality, we assume that the discontinuity is located at $\left[x_{i+1}, x_{i+2}\right]$. Similar to the analysis in Section 3.1, we can obtain the values of the parameters $r_{1}$ and $r_{2}$ in Equation (38) as follows:

$$
r_{1}=\left\{\begin{array}{ll}
2, & f_{i}^{\prime} \neq 0, \\
4, & f_{i}^{\prime}=0,
\end{array} \quad r_{2}= \begin{cases}4, & f_{i}^{\prime} \neq 0, \\
2, & f_{i}^{\prime}=0 .\end{cases}\right.
$$

Compared with Equations (40) and (42), it is found that the value of $r_{2}$ is doubled. Finally, we have

$$
\omega_{k}=\left\{\begin{array}{ll}
c_{k}^{4,0}+O\left(\Delta x^{2}\right), & f_{i}^{\prime} \neq 0, \\
c_{k}^{4,0}+O\left(\Delta x^{2}\right), & f_{i}^{\prime}=0,
\end{array} \quad k=0,1, \omega_{2} \rightarrow 0 .\right.
$$

Similarly, results can be obtained for the case of the discontinuity located at $\left[x_{i-2}, x_{i-1}\right]$. Numerical examples show that this improvement can effectively reduce the dissipation near the discontinuities.

In addition, if the discontinuity is located at $\left[x_{i-1}, x_{i}\right]$ and $\left[x_{i}, x_{i+1}\right]$, the weights of the present scheme are approximated by $\omega_{0} \rightarrow 0, \omega_{1} \rightarrow 0, \omega_{2} \rightarrow 1$, and $\omega_{0} \rightarrow 1, \omega_{1} \rightarrow 0, \omega_{2} \rightarrow 0$, respectively. Therefore, the new WENO scheme can keep the ENO property near shock waves.

\section{4 | NUMERICAL EXAMPLES}

In this section, several numerical examples are given to compare the following schemes:

(1) WENO-Z: Weighting method Equation (12) with $q=1$ and $\varepsilon=10^{-40}$;

(2) WENO-Z2: Weighting method Equation (12) with $q=2$ and $\varepsilon=10^{-40}$;

(3) WENO-Zdx3 $3^{22}$ : Weighting method Equation (12) with $q=1$ and $\varepsilon=\Delta x^{3}$;

(4) WENO-Z2dx4 $4^{22}$ : Weighting method Equation (12) with $q=2$ and $\varepsilon=\Delta x^{4}$;

(5) M-WENO: Multistep WENO scheme Equation (23) with $\varepsilon=10^{-40}$;

(6) E-WENO: Embedded WENO-Z scheme Equation (27) with $\varepsilon=10^{-40}$;

(7) IM-WENO: Improved multistep WENO scheme Equation (28) with $\varepsilon=10^{-40}$;

(8) IM-WENO-2: IM-WENO scheme with $q=2$ and $\varepsilon=10^{-40}$;

(9) HM-WENO: New WENO scheme Equations (45) and (46) with $\varepsilon=10^{-40}$;

(10) HM-WENO-2: New WENO scheme Equations (47) and (48) with $\varepsilon=10^{-40}$.

The fourth-order Runge-Kutta method ${ }^{24}$ is used to approximate the time derivative, and the CFL number is set to be 0.5 for all examples in this paper.

\subsection{The accuracy at critical point}

Referring to the works of Castro et $\mathrm{al}^{7}$ and Fan et al, ${ }^{9}$ the function $f(x)=x^{2} e^{x}$, which has a critical point at $x=0$ where $f^{\prime}(0)=0$ and $f^{\prime \prime}(0) \neq 0$, is also used to measure the accuracy of the new scheme and several WENO schemes mentioned 
TABLE 2 Comparison of errors and convergence orders for different schemes at critical point

\begin{tabular}{|cccccccccccc}
\hline $\boldsymbol{\Delta} \boldsymbol{x}$ & \multicolumn{2}{c}{ WENO-Z } & \multicolumn{2}{c}{ WENO-Zdx3 } & \multicolumn{2}{c}{ M-WENO } & \multicolumn{2}{c}{ IM-WENO } & \multicolumn{2}{c}{ HM-WENO } \\
& Error & order & Error & order & Error & order & Error & order & Error & order \\
\hline $2 \mathrm{e}-2$ & $0.496 \mathrm{e}-06$ & - & $0.844 \mathrm{e}-08$ & - & $0.154 \mathrm{e}-08$ & - & $0.286 \mathrm{e}-06$ & - & $0.265 \mathrm{e}-07$ & - \\
\hline $1 \mathrm{e}-2$ & $0.292 \mathrm{e}-07$ & 4.09 & $0.192 \mathrm{e}-09$ & 5.46 & $0.496 \mathrm{e}-10$ & 4.95 & $0.159 \mathrm{e}-07$ & 4.17 & $0.968 \mathrm{e}-09$ & 4.78 \\
\hline $5 \mathrm{e}-3$ & $0.176 \mathrm{e}-08$ & 4.05 & $0.411 \mathrm{e}-11$ & 5.55 & $0.156 \mathrm{e}-11$ & 4.99 & $0.923 \mathrm{e}-09$ & 4.11 & $0.326 \mathrm{e}-10$ & 4.89 \\
\hline $2.5 \mathrm{e}-3$ & $0.108 \mathrm{e}-09$ & 4.03 & $0.915 \mathrm{e}-13$ & 5.49 & $0.488 \mathrm{e}-13$ & 5.00 & $0.552 \mathrm{e}-10$ & 4.06 & $0.106 \mathrm{e}-11$ & 4.94 \\
\hline $1.25 \mathrm{e}-3$ & $0.666 \mathrm{e}-11$ & 4.02 & $0.222 \mathrm{e}-14$ & 5.37 & $0.152 \mathrm{e}-14$ & 5.00 & $0.337 \mathrm{e}-11$ & 4.03 & $0.338 \mathrm{e}-13$ & 4.97 \\
\hline $\boldsymbol{\Delta} \boldsymbol{x}$ & $\mathbf{W E N O}-Z 2$ & WENO-Z2dx4 & E-WENO & IM-WENO-2 & HM-WENO-2 \\
\hline $2 \mathrm{e}-2$ & $0.256 \mathrm{e}-06$ & - & $0.127 \mathrm{e}-06$ & - & $0.138 \mathrm{e}-05$ & - & $0.194 \mathrm{e}-06$ & - & $0.461 \mathrm{e}-08$ & - \\
\hline $1 \mathrm{e}-2$ & $0.579 \mathrm{e}-08$ & 5.47 & $0.323 \mathrm{e}-08$ & 5.30 & $0.817 \mathrm{e}-07$ & 4.08 & $0.397 \mathrm{e}-08$ & 5.61 & $0.613 \mathrm{e}-10$ & 6.23 \\
\hline $5 \mathrm{e}-3$ & $0.144 \mathrm{e}-09$ & 5.33 & $0.888 \mathrm{e}-10$ & 5.19 & $0.500 \mathrm{e}-08$ & 4.03 & $0.894 \mathrm{e}-10$ & 5.47 & $0.157 \mathrm{e}-11$ & 5.28 \\
\hline $2.5 \mathrm{e}-3$ & $0.392 \mathrm{e}-11$ & 5.20 & $0.258 \mathrm{e}-11$ & 5.10 & $0.310 \mathrm{e}-09$ & 4.01 & $0.224 \mathrm{e}-11$ & 5.32 & $0.482 \mathrm{e}-13$ & 5.03 \\
\hline $1.25 \mathrm{e}-3$ & $0.113 \mathrm{e}-12$ & 5.11 & $0.776 \mathrm{e}-13$ & 5.06 & $0.193 \mathrm{e}-10$ & 4.01 & $0.615 \mathrm{e}-13$ & 5.19 & $0.151 \mathrm{e}-14$ & 4.99 \\
\hline
\end{tabular}

above. Meanwhile, in order to evaluate the influence of the value of $\varepsilon$ on the accuracy of WENO scheme at critical point, $\varepsilon$ is taken as the power of $\Delta x$ employed in the work of Don and Borges ${ }^{22}$ and a fixed constant $10^{-40}$ recommended by Henrick et al, ${ }^{5}$ respectively. Table 2 gives the comparison of the errors of the first derivative of the function $f(x)$ at $x=0$ and convergence orders of different schemes. The critical point is set as a grid point $x_{i}$ and the flux $\hat{f}_{i+1 / 2}$ is calculated by using the five-point stencil of $\left[x_{i-2}, x_{i-1}, \ldots, x_{i+2}\right]$, where, $x_{i}=0, x_{i \pm n}=x_{i} \pm n \Delta x$. From this table, we can see that WENO-Zdx3 and WENO-Z2dx4, ${ }^{22} \mathrm{M}$-WENO,${ }^{16}$ and HM-WENO achieve fifth-order accuracy at critical point. Hence, as analyzed in the work of Don and Borges, ${ }^{22}$ the WENO-Z scheme can obtain the optimal convergence order at critical point if $\varepsilon$ takes the power of $\Delta x$. The IM-WENO scheme ${ }^{19}$ has the same fourth-order accuracy as the WENO-Z scheme $\left(q=1, \varepsilon=10^{-40}\right)$, although its error is less than WENO-Z's. For the case of the power parameter $q=2$, the E-WENO scheme is only fourth-order accuracy. As analyzed in Section 3.1, the E-WENO scheme decreases the accuracy of the WENO-Z scheme (with $q=2$ ).

\subsection{The errors around transition point}

In this subsection, the following function

$$
f(x)=\left\{\begin{array}{lr}
A \sin (k \pi x), & -1 \leq x \leq 0, \\
A \sin (k \pi x)+1, & 0<x \leq 1
\end{array}\right.
$$

is used to measure the performance of different schemes mentioned above around transition point, where $A=0.01, k=6$. For this case, $x_{i}=0$ and the next point $x_{i+1}$ are the discontinuity points. Hence, the points $x_{i-1}$ and $x_{i+3}$ are the transition points. Table 3 gives the comparison of errors of first-order derivative of $f(x)$ in Equation (58) for different schemes around the transition points with $N=80, N=160$, and $N=320$, and the transition points are displayed in bold. It can be seen that the errors of the multistep WENO-type schemes (M-WENO, IM-WENO, IM-WENO-2, E-WENO, HM-WENO, HM-WENO-2) are less than those of WENO-Z-type schemes (WENO-Z, WENO-Z2, WENO-Zdx3, WENO-Z2dx4) at transition points.

\section{3 | One-dimensional linear advection problems}

The governing equation of linear advection problems is given by

$$
\left\{\begin{array}{l}
\frac{\partial u}{\partial t}+\frac{\partial u}{\partial x}=0, \\
u(x, 0)=u_{0}(x) .
\end{array}\right.
$$

The periodic boundary condition is applied for this kind of problem. 


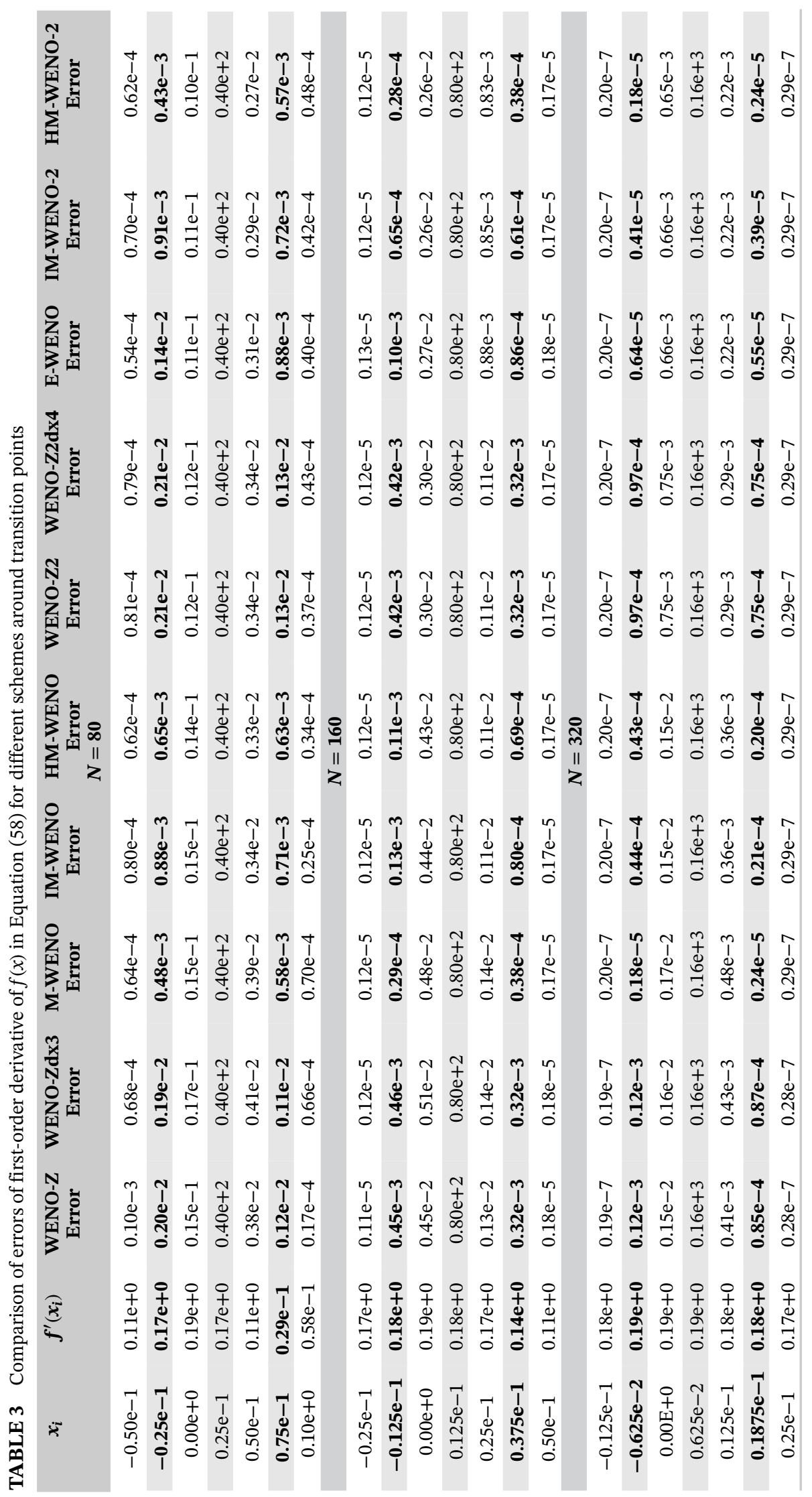




\subsection{1 | The first case of advection equation}

The initial condition is given by

$$
u_{0}(x)= \begin{cases}\frac{1}{6}(G(x, \beta, z-\delta)+G(x, \beta, z+\delta)+4 G(x, \beta, z)), & -0.8 \leqslant x \leqslant-0.6 \\ 1, & -0.4 \leqslant x \leqslant-0.2 \\ 1-|10(x-0.1)|, & 0 \leqslant x \leqslant 0.2 \\ \frac{1}{6}(F(x, \alpha, a-\delta)+F(x, \alpha, a+\delta)+4 F(x, \alpha, a)), & 0.4 \leqslant x \leqslant 0.6 \\ 0, & \text { otherwise, }\end{cases}
$$

with the computational domain $[-1,1]$, where $G(x, \beta, z)=e^{-\beta(x-z)^{2}}, F(x, \alpha, a)=\sqrt{\max \left(1-\alpha^{2}(x-a)^{2}, 0\right)}, a=0.5, z=$ $-0.7, \delta=0.005, \alpha=10$, and $\beta=\log 2 / 36 \delta^{2}$. The solution contains a Gaussian, a square-wave, a triangle, and a semiellipse wave. Figure 2 gives the numerical results of different schemes. From the enlarged part of this figure, we can find that the
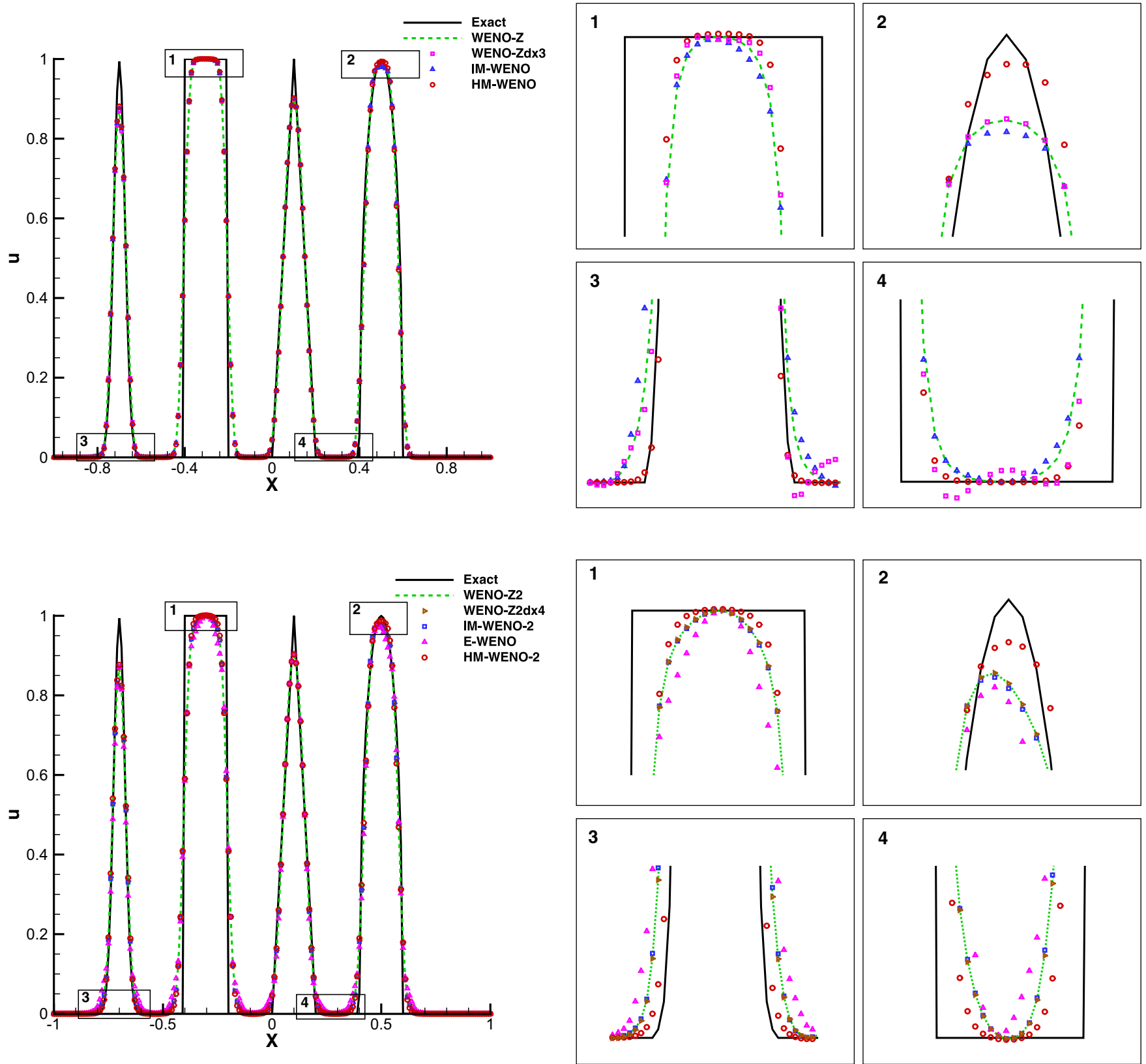

FIGURE 2 Linear advection problem with initial condition Equation (60) at $t=6, N=200$ [Colour figure can be viewed at wileyonlinelibrary.com] 


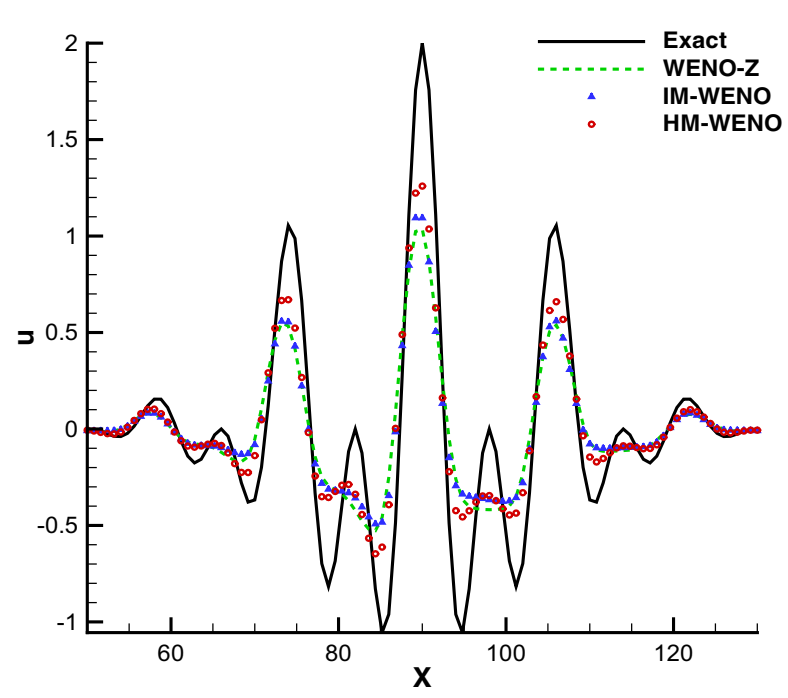

(A)

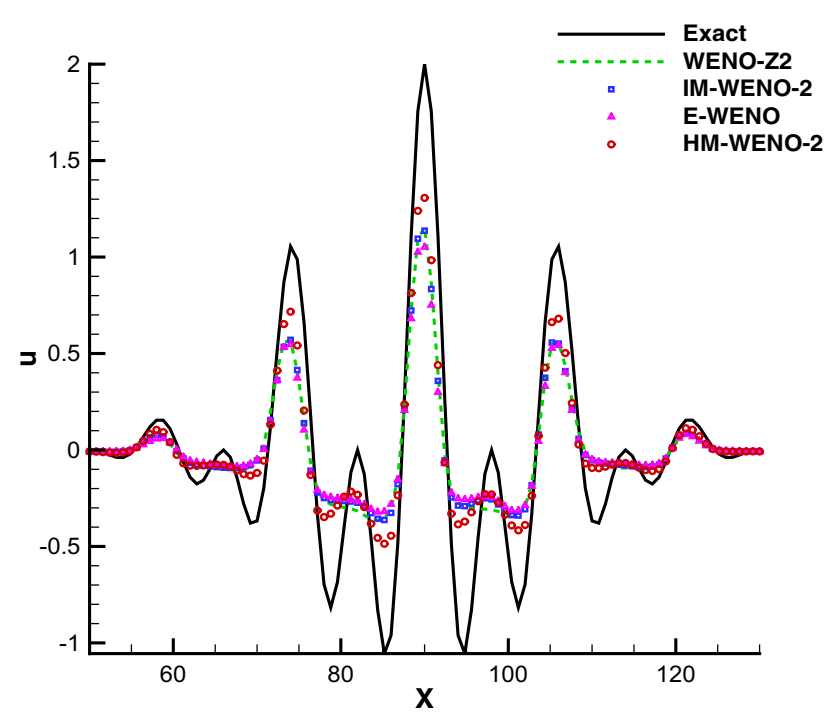

(B)

FIGURE 3 Linear advection problem with initial condition Equation (61) with $N=100$ at (A) $t=960$, (B) $t=400$ [Colour figure can be viewed at wileyonlinelibrary.com]

IM-WENO and E-WENO schemes even generate less accurate solution than WENO-Z with $q=1$ and $q=2$, respectively. The HM-WENO and HM-WENO-2 schemes can effectively reduce the dissipation near the discontinuities, and they have the best resolution for four kinds of waves.

Apparently, as shown in Section 4.1 and in the work of Don and Borges, ${ }^{22}$ the WENO-Z (with $\varepsilon=\Omega\left(\Delta x^{m}\right)$ ) scheme can obtain the optimal convergence order at critical points (even at high-order critical points). However, as stated in the work of Don and Borges, ${ }^{22}$ for $q=1$, both the WENO-JS and WENO-Z schemes do not have sufficient numerical dissipation to remove the numerical oscillations from the solution, regardless of the order of schemes and $\varepsilon$ chosen. Since this paper mainly focuses on the improvement at transition point, for the rest examples, only the case of $\varepsilon=10^{-40}$ is discussed and compared, although the improvement strategy is still available for those WENO schemes with $\varepsilon=\Omega\left(\Delta x^{m}\right)$.

\subsubsection{The second case of advection equation}

The initial condition is given by

$$
u_{0}(x)=e^{-(x-90)^{2} / 400}\left(\cos \left(\frac{\pi}{8}(x-90)\right)+\cos \left(\frac{\pi}{4}(x-90)\right)\right), 50 \leqslant x \leqslant 130 .
$$

Figure 3 gives the numerical results of different schemes. Similar to the above example, the HM-WENO and HM-WENO-2 schemes apparently improve the accuracy of the solution of the high-frequency wave problem.

Since the comparison and conclusion for those schemes with $q=2$ are in agreement with those with $q=1$, in the rest of this paper, only the results of the WENO-Z, IM-WENO, and HM-WENO schemes are shown and discussed.

\subsubsection{The third case of advection equation}

The initial condition is given by ${ }^{5}$

$$
u_{0}(x)=\sin \left(\pi x-\frac{\sin (\pi x)}{\pi}\right),-1 \leqslant x \leqslant 1 .
$$

This solution has two critical points, where $u^{\prime}(0)=0$ and $u^{\prime \prime \prime}(0) \neq 0$. As in the works of Jiang and Shu ${ }^{2}$ and Ha et al, ${ }^{8}$ the time step is set to $\Delta x^{5 / 4}$. The program with quadruple precision is performed on Intel Xeon E5-2640 v3. Table 4 gives the errors, convergence orders, and CPU time of different schemes at $t=2$. From this table, it can be seen that the HM-WENO scheme gets the same fifth-order accuracy as the first multistep WENO (M-WENO) scheme. While the convergence orders of the WENO-Z and IM-WENO schemes gradually decrease to fourth order with refined meshes. The comparisons of computational efficiency are shown in Figure 4. As observed from this figure, the HM-WENO scheme greatly improves the computational efficiency of the M-WENO scheme. It can be seen that HM-WENO costs about $30 \%$ and $15 \%$ CPU time more than WENO-Z and IM-WENO, respectively; however, if a more accurate solution (with a refined mesh) is required, the HM-WENO scheme is more efficient than both the WENO-Z and IM-WENO schemes. 
TABLE 4 Comparison of errors, convergence orders, and CPU time (in seconds) for different schemes

\begin{tabular}{ccccccccc}
$\boldsymbol{N}$ & \multicolumn{2}{c}{ WENO-Z } & \multicolumn{2}{c}{ IM-WENO } & \multicolumn{2}{c}{ M-WENO } & \multicolumn{2}{c}{ HM-WENO } \\
& $\boldsymbol{L}_{\infty}$ (order) & CPU Time & $\boldsymbol{L}_{\infty}$ (order) & CPU time & $\boldsymbol{L}_{\infty}$ (order) & CPU Time & $\boldsymbol{L}_{\infty}$ (order) & CPU Time \\
\hline 160 & $0.21 \mathrm{e}-06(-)$ & $7.0 \mathrm{e}-01$ & $0.21 \mathrm{e}-06(-)$ & $7.6 \mathrm{e}-01$ & $0.21 \mathrm{e}-06(-)$ & $1.6 \mathrm{e}+00$ & $0.21 \mathrm{e}-06(-)$ & $8.9 \mathrm{e}-01$ \\
\hline 320 & $0.78 \mathrm{e}-08(4.76)$ & $3.3 \mathrm{e}+00$ & $0.66 \mathrm{e}-08(5.00)$ & $3.5 \mathrm{e}+00$ & $0.66 \mathrm{e}-08(5.00)$ & $7.3 \mathrm{e}+00$ & $0.66-08(5.00)$ & $4.1 \mathrm{e}+00$ \\
\hline 640 & $0.36 \mathrm{e}-09(4.44)$ & $1.5 \mathrm{e}+01$ & $0.20 \mathrm{e}-09(5.00)$ & $1.7 \mathrm{e}+01$ & $0.20 \mathrm{e}-09(5.00)$ & $3.5 \mathrm{e}+01$ & $0.20 \mathrm{e}-09(5.00)$ & $1.9 \mathrm{e}+01$ \\
\hline 1280 & $0.17 \mathrm{e}-10(4.37)$ & $7.3 \mathrm{e}+01$ & $0.84 \mathrm{e}-11(4.61)$ & $7.8 \mathrm{e}+01$ & $0.64 \mathrm{e}-11(5.00)$ & $1.6 \mathrm{e}+02$ & $0.64 \mathrm{e}-11(5.00)$ & $9.1 \mathrm{e}+01$ \\
2560 & $0.82 \mathrm{e}-12(4.39)$ & $3.4 \mathrm{e}+02$ & $0.40 \mathrm{e}-12(4.39)$ & $3.7 \mathrm{e}+02$ & $0.20 \mathrm{e}-12(5.00)$ & $7.7 \mathrm{e}+02$ & $0.20 \mathrm{e}-12(5.00)$ & $4.3 \mathrm{e}+02$ \\
\hline
\end{tabular}

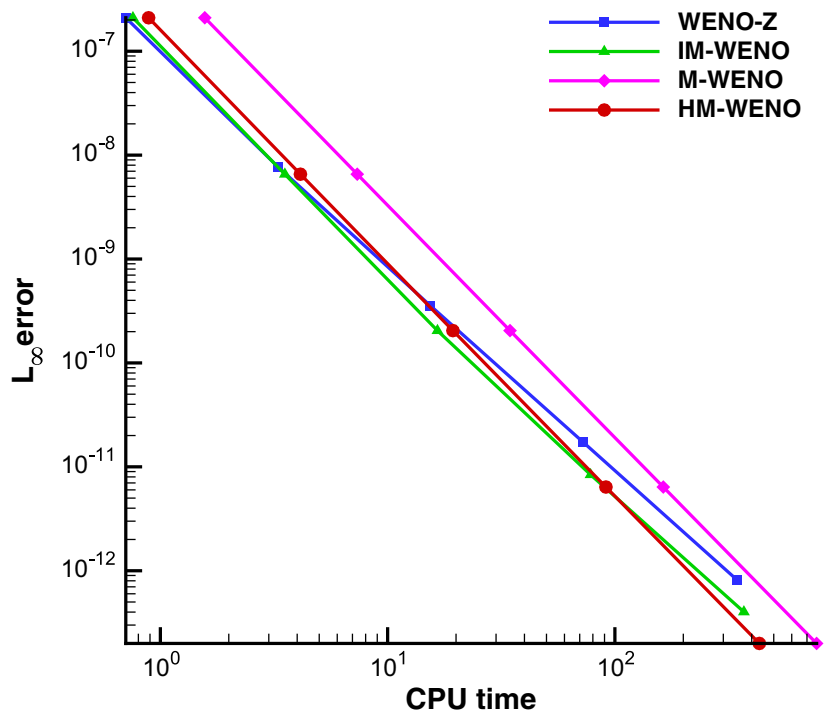

FIGURE 4 Comparisons of computational efficiency [Colour figure can be viewed at wileyonlinelibrary.com]

\section{4 | One-dimensional Euler problems}

The governing equation of one-dimensional Euler problems is given by

$$
\frac{\partial U}{\partial t}+\frac{\partial F(U)}{\partial x}=0
$$

where $U=(\rho, \rho u, E)^{T}, F(U)=\left(\rho u, \rho u^{2}+p, u(E+p)\right)^{T} ; \rho, u, E, p$ denote the density, velocity, total energy, and pressure, respectively, and for ideal gas $E=\frac{p}{\gamma-1}+\frac{1}{2} \rho u^{2} \cdot \gamma=1.4$ is the ratio of specific heat. The global Lax-Friedrichs flux-splitting method with local characteristic reconstruction is used for the inviscid flux.

\subsection{1 | Lax problem}

The initial conditions of this problem are

$$
(\rho, u, p)=\left\{\begin{array}{lr}
(0.445,0.698,3.528), & -5 \leq x<0 \\
(0.5,0,0.571), & 0 \leq x \leq 5,
\end{array}\right.
$$

with zero gradient boundary conditions at $x= \pm 5$. Figure 5 gives the numerical results with $N=200$ at $t=1.3$. The reference result is obtained by the WENO-Z scheme with refined grids. From the enlarged part of this figure, we can see that the present scheme is more accurate than the other schemes near shock waves.

\subsection{2 | Shock entropy wave interaction}

The initial conditions of this problem ${ }^{25}$ are

$$
(\rho, u, p)= \begin{cases}(3.857143,2.629369,31 / 3), & -5 \leq x<-4 \\ (1+0.2 \sin (5 x), 0,1), & -4 \leq x \leq 5,\end{cases}
$$




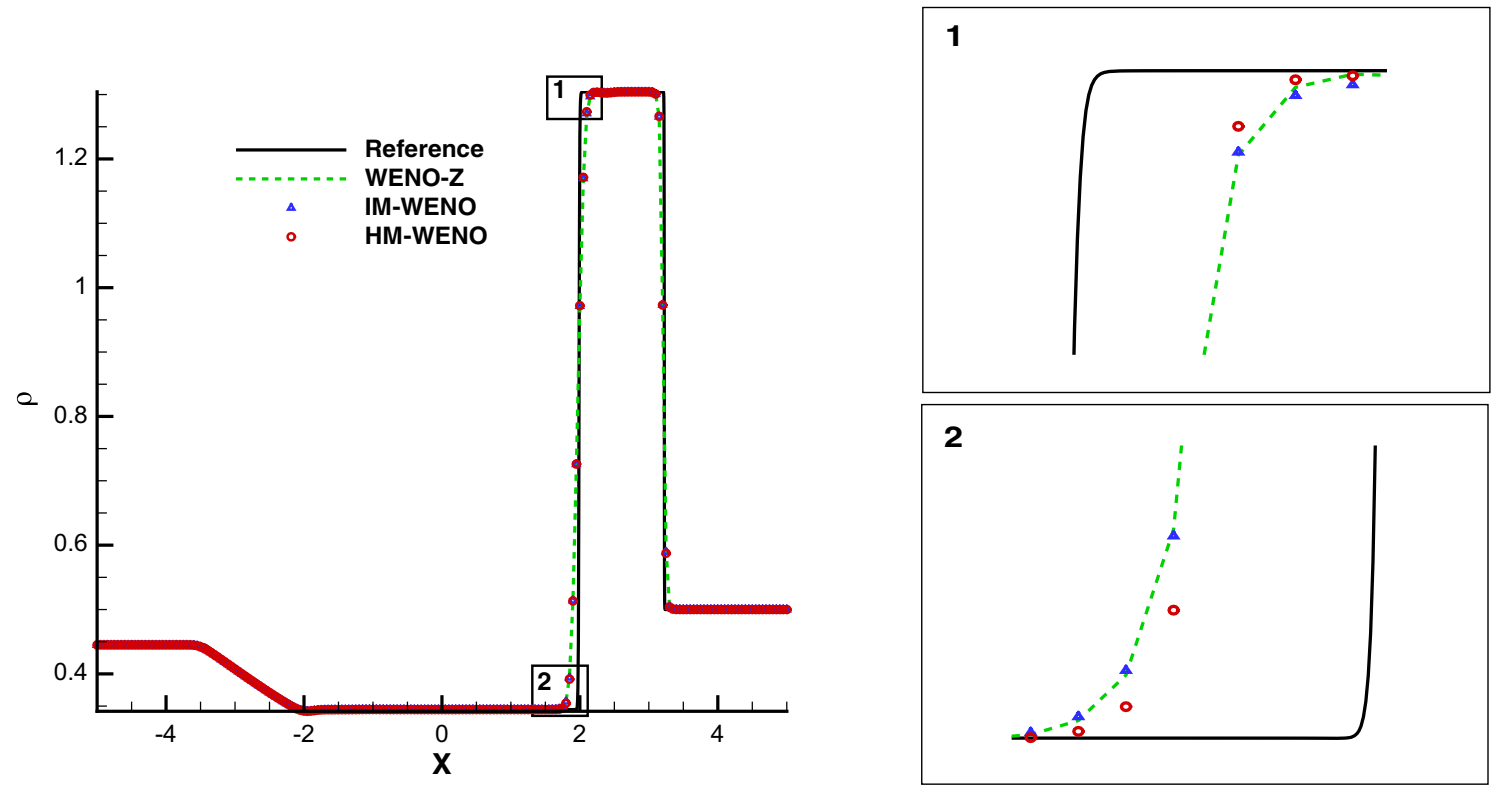

FIGURE 5 Lax problem at $t=1.3, N=200$ [Colour figure can be viewed at wileyonlinelibrary.com]
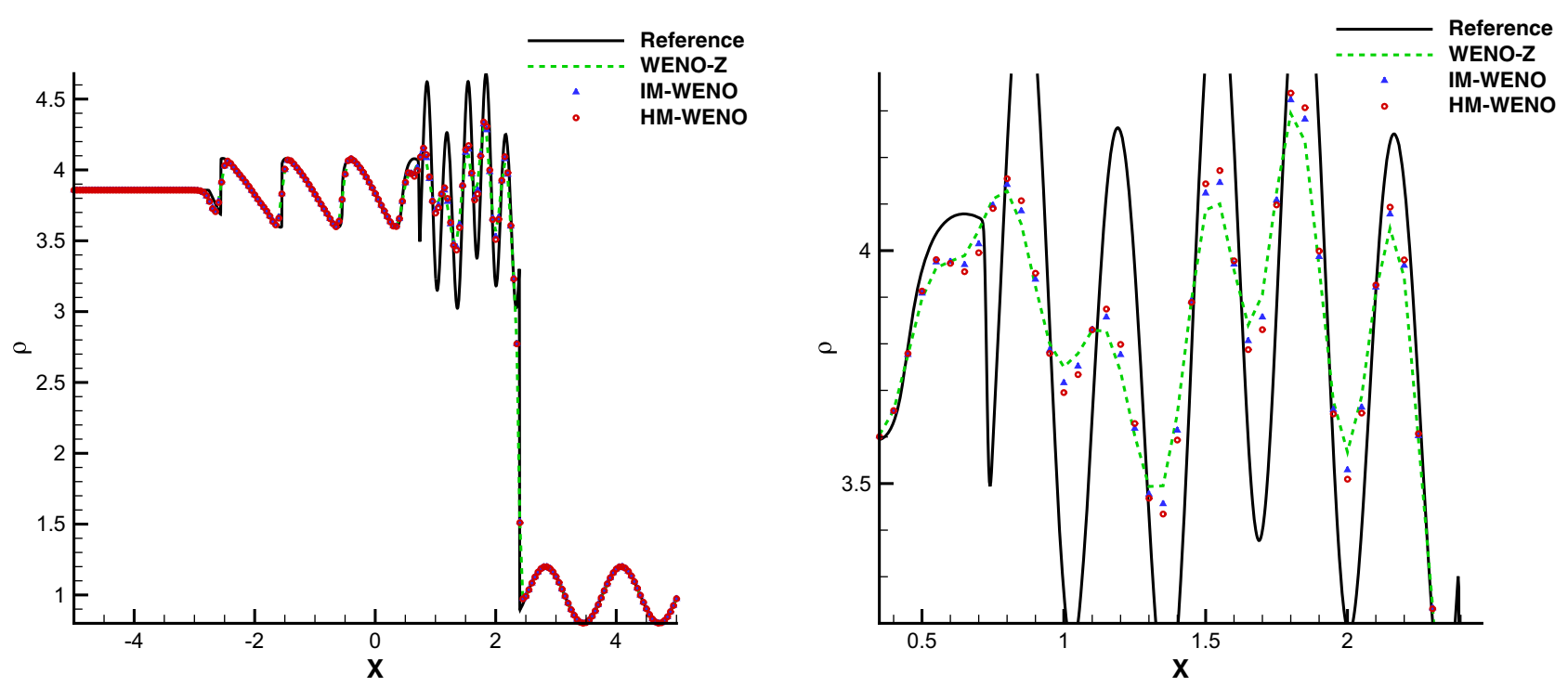

FIGURE 6 Shock entropy wave interaction at $t=1.8, N=200$ [Colour figure can be viewed at wileyonlinelibrary.com]

with zero gradient boundary conditions at $x= \pm 5$. This case represents a Mach 3 shock wave interacting with a sine entropy wave and is widely used for evaluating the resolution of the shock-capturing schemes. The numerical results of different schemes with $N=200$ at $t=1.8$ are shown in Figure 6 . This example shows that, for the high frequency waves, the HM-WENO scheme can also obtain more accurate results than the WENO-Z and IM-WENO schemes.

\subsubsection{Interacting blast waves}

The initial conditions of this problem ${ }^{5,6}$ are

$$
(\rho, u, p)= \begin{cases}(1,0,1000), & 0 \leq x<0.1 \\ (1,0,0.01), & 0.1 \leq x<0.9 \\ (1,0,100), & 0.9 \leq x \leq 1\end{cases}
$$



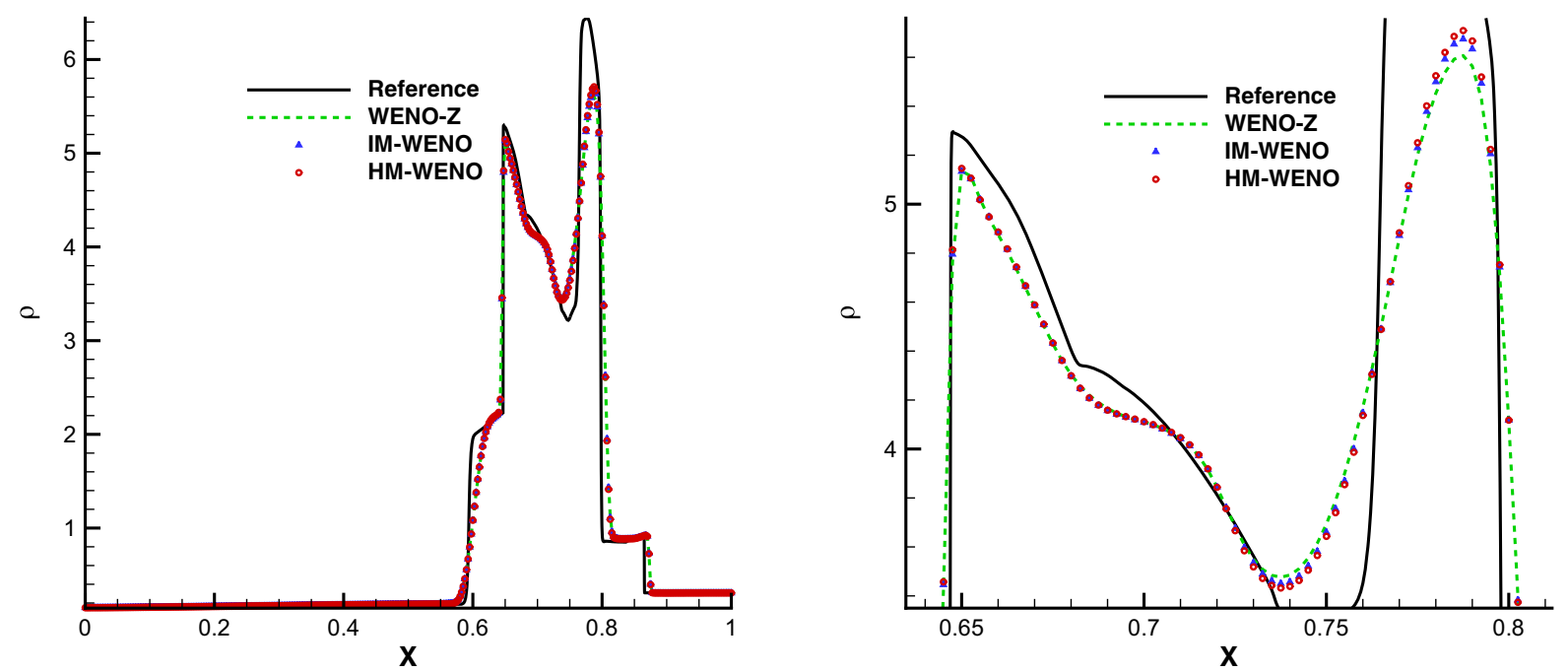

FIGURE 7 Interacting blast waves at $t=0.038, N=400$ [Colour figure can be viewed at wileyonlinelibrary.com]

with reflection boundary conditions at $x=0$ and $x=1$. This case is often used as a benchmark case to test the robustness and the capability of shock-capturing for different schemes. Figure 7 gives the numerical results with $N=400$ at $t=0.038$. It can be observed that the present scheme can capture the strong shock as well as the WENO-Z and IM-WENO schemes.

\section{5 | 2D Euler problems}

The governing equation of 2D Euler problems is given by

$$
\frac{\partial U}{\partial t}+\frac{\partial F}{\partial x}+\frac{\partial G}{\partial y}=0
$$

where

$$
U=\left[\begin{array}{c}
\rho \\
\rho u \\
\rho v \\
E
\end{array}\right], \quad F=\left[\begin{array}{c}
\rho u \\
\rho u^{2}+p \\
\rho u v \\
E u+p u
\end{array}\right], \quad G=\left[\begin{array}{c}
\rho v \\
\rho u v \\
\rho v^{2}+p \\
E v+p v
\end{array}\right] .
$$

The specific total energy $E$ is given by

$$
E=\frac{p}{(\gamma-1)}+\frac{1}{2} \rho\left(u^{2}+v^{2}\right)
$$

The Steger-Warming flux splitting method ${ }^{26}$ is used for the inviscid convective fluxes. The WENO reconstruction is carried out for each component of the split fluxes. The time step is taken $\operatorname{as}^{27}$

$$
\Delta t=C F L \frac{\Delta t_{x} \Delta t_{y}}{\Delta t_{x}+\Delta t_{y}}, \text { with } \Delta t_{x}=\frac{\Delta x}{\max _{i, j}\left(\left|u_{i, j}\right|+c_{i, j}\right)} \text { and } \Delta t_{y}=\frac{\Delta y}{\max _{i, j}\left(\left|v_{i, j}\right|+c_{i, j}\right)},
$$

where $c$ is the speed of sound.

\subsection{1 | 2D vortex evolution problem}

The $2 \mathrm{D}$ vortex evolution problem ${ }^{28}$ is often used to evaluate the dissipation property of a scheme. It describes an isentropic vortex moves across the computational domain periodically. The initial conditions of this problem are

$$
\left\{\begin{array}{l}
u=0.5-\frac{\varepsilon e^{\left(1-r^{2}\right) / 2}}{2 \pi}(y-5) \\
v=\frac{\varepsilon e^{\left(1-r^{2}\right) / 2}}{2 \pi}(x-5) \\
T=1-\frac{(\gamma-1) \varepsilon^{2} e^{\left(1-r^{2}\right)}}{8 \gamma \pi^{2}} \\
S=1
\end{array}\right.
$$




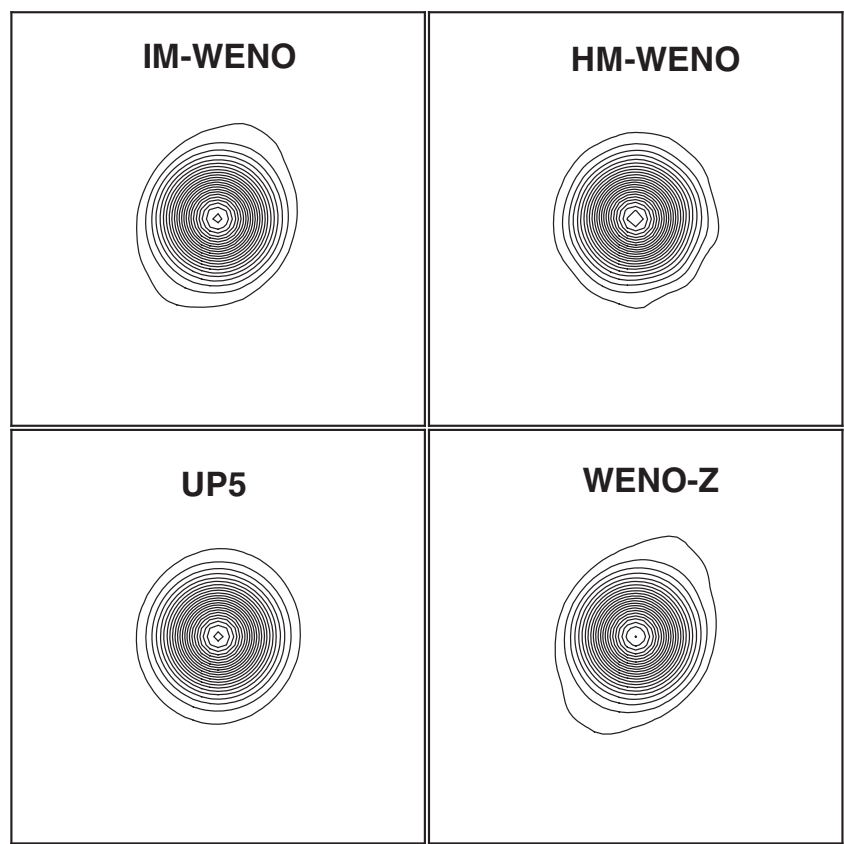

FIGURE 8 Pressure contours of the vortex evolution problem, 30 contours from 0.9918 to 0.9998

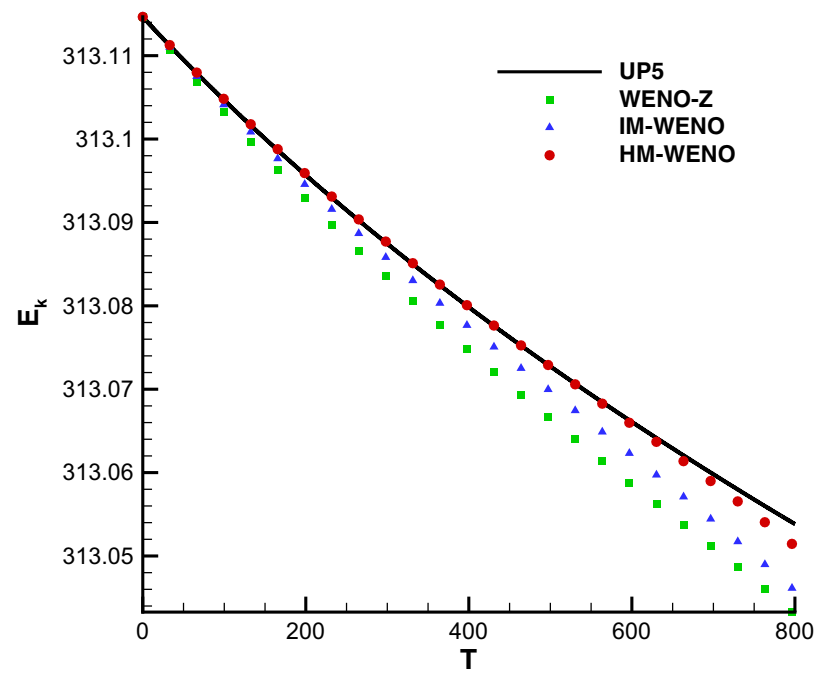

FIGURE 9 Integrated kinetic energy versus time [Colour figure can be viewed at wileyonlinelibrary.com]

The temperature $T$ and entropy $S$ are defined as follows:

$$
T=\frac{p}{\rho}, S=\frac{p}{\rho^{\gamma}},
$$

where $r^{2}=(x-5)^{2}+(y-5)^{2}$, and vortex strength $\varepsilon$ is 0.5 . The computational domain is $[0,10] \times[0,10]$. Periodic boundary conditions are set for all boundaries. Figure 8 gives the pressure contours of different schemes with $N_{x} \times N_{y}=50 \times 50$ at $t=800$. The evolution of the integrated kinetic energy $\left(E_{k}=\sum_{i, j} \rho_{i, j}\left(u_{i, j}^{2}+v_{i, j}^{2}\right) / 2\right)$ is shown in Figure 9 . It can be seen that the HM-WENO scheme maintains the symmetry of the vortex structure better than the WENO-Z and IM-WENO schemes, and its results are close to those of the fifth-order upstream scheme (UP5). 


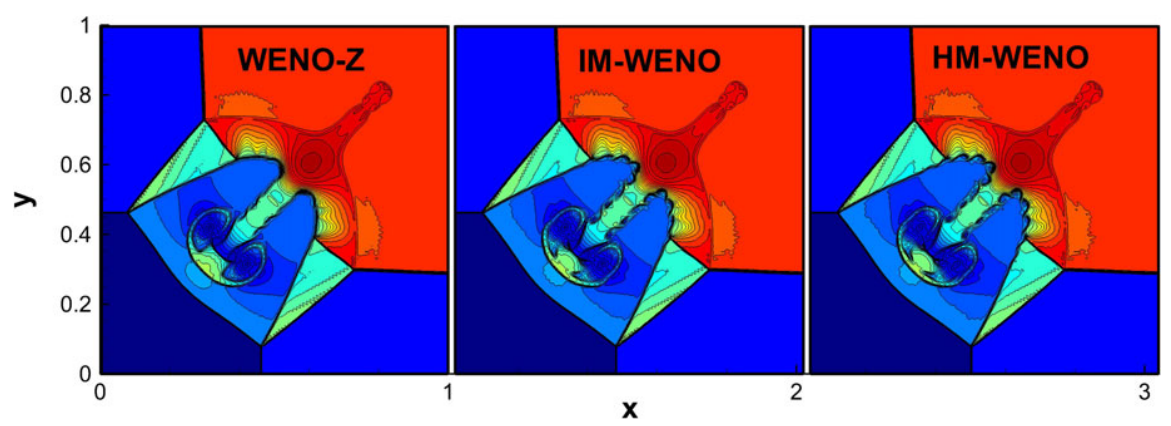

FIGURE 10 Two-dimensional Riemann problem, $N_{x} \times N_{y}=400 \times 400,25$ contours from 0.2 to 1.85 [Colour figure can be viewed at wileyonlinelibrary.com]

\subsection{2 | 2D Riemann problem}

The initial conditions of this problem ${ }^{19,29}$ are

$$
(\rho, u, v, p)= \begin{cases}(1.5,0,0,1.5), & 0.8 \leq x \leq 1,0.8 \leq y \leq 1 \\ (0.5323,1.206,0,0.3), & 0 \leq x<0.8,0.8 \leq y \leq 1 \\ (0.138,1.206,1.206,0.029), & 0 \leq x<0.8,0 \leq y<0.8 \\ (0.5323,0,1.206,0.3), & 0.8 \leq x \leq 1,0 \leq y<0.8,\end{cases}
$$

with Dirichlet boundary conditions. The computational domain is $[0,1] \times[0,1]$. Figure 10 gives the density contours with $N_{x} \times N_{y}=400 \times 400$ at $t=0.8$. It can be found that all schemes can capture reflection shocks and contact discontinuities well. Comparing the structures caused by Kelvin-Helmholtz instabilities, it can be seen that HM-WENO generates richer structures than IM-WENO and WENO-Z, which means the new scheme has less numerical dissipation near contact interfaces.

\subsection{3 | Double Mach reflection problem}

This problem is proposed by Woodward and Colella ${ }^{30}$ and describes the reflection of a planar Mach shock in air hitting a wedge. The initial conditions of this problem are

$$
(\rho, u, v, p)= \begin{cases}\left(8,8.25 \cos \frac{\pi}{6},-8.25 \sin \frac{\pi}{6}, 116.5\right), & x<\frac{1}{6}+\frac{y}{\sqrt{3}} \\ (1.4,0,0,1.0), & x \geqslant \frac{1}{6}+\frac{y}{\sqrt{3}}\end{cases}
$$

The computational domain is $[0,4] \times[0,1]$. For the bottom boundary, the exact post-shock condition is imposed for the interval $[0,0.6]$, and a reflective boundary condition is used for the rest. The top boundary is set to describe the exact motion of a Mach 10 shock. Inflow and outflow boundary conditions are used for the left and right boundaries, respectively. Figure 11 gives the density contours with $N_{x} \times N_{y}=960 \times 240$ at $t=0.2$. Meanwhile, the magnification of the blow-up region around the double Mach stems of each plot is also shown in the picture. It can be found that all schemes can capture shock structures well. However, from three enlarged plots, we can see that the HM-WENO scheme resolves more instability structures than the other two schemes.

\subsection{4 । Forward-facing step problem}

This problem is also originally from the work of Woodward and Colella. ${ }^{30}$ The setup of the problem is as follows: the wind tunnel spans a domain of $[0,3] \times[0,1]$. A forward-facing step is located at $(0.6,0.2)$. The problem is initialized by a right-going Mach 3 flow with a density of 1.4 and a pressure of unity. Reflective boundary conditions are applied along the walls of the tunnel. Inflow and outflow boundary conditions are used for the left and right boundaries, respectively. The density contours with $N_{x} \times N_{y}=600 \times 200$ at $t=4$ is shown in Figure 12. All the schemes perform well with good resolution. Carefully comparing these figures, it can be observed that the roll-up of the vortex sheet captured by HM-WENO is more clear than the others. 


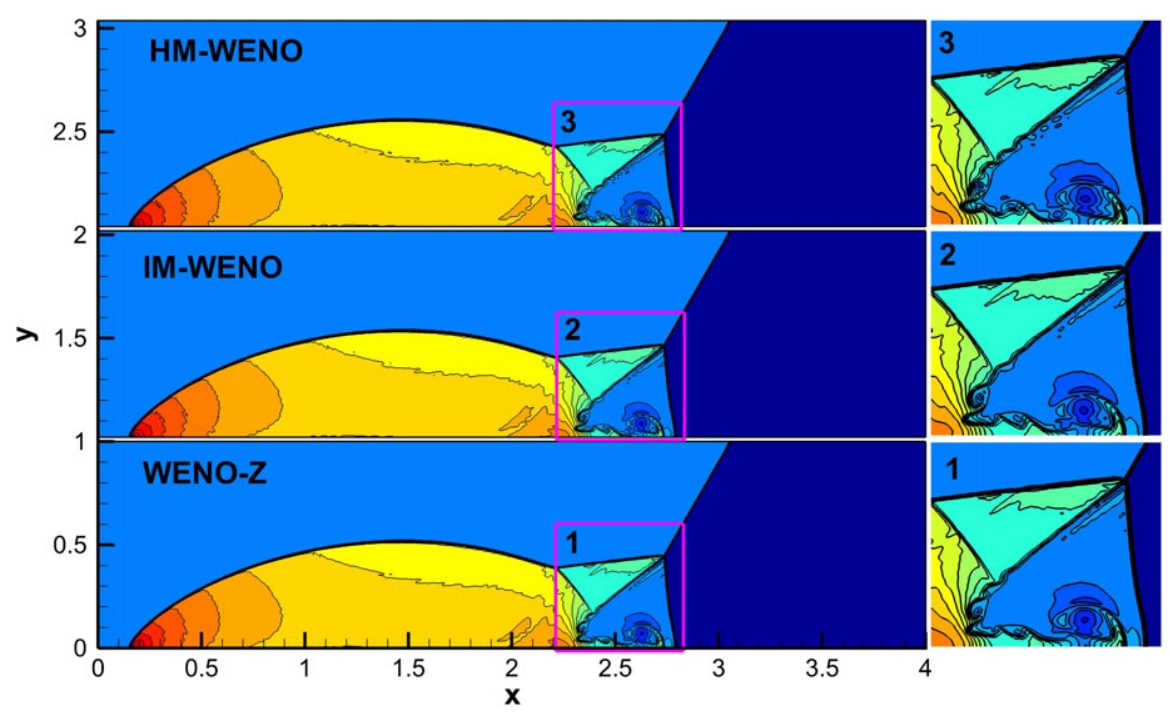

FIGURE 11 Double Mach reflection problem, $N_{x} \times N_{y}=960 \times 240,30$ contours from 1.2 to 24 [Colour figure can be viewed at wileyonlinelibrary.com]

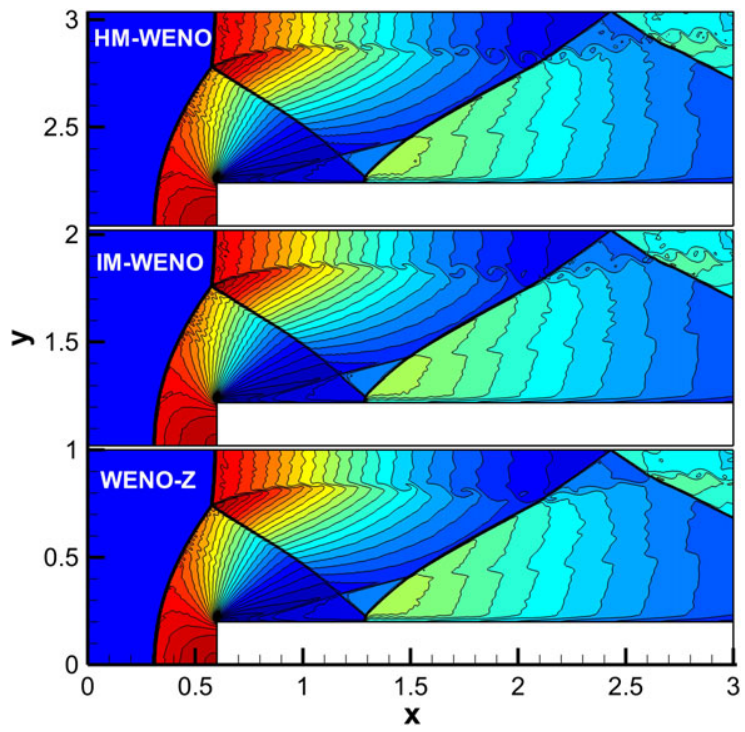

FIGURE 12 Forward-facing step problem, $N_{x} \times N_{y}=600 \times 200,30$ contours from 0.3 to 6.4 [Colour figure can be viewed at wileyonlinelibrary.com]

\subsection{5 | 2D Sedov blast wave problem}

The 2D Sedov blast wave problem, ${ }^{31,32}$ which has an exact self-similar solution, is a well-known benchmark test to study a strong explosion problem. The initial conditions of this problem are

$$
(\rho, u, v, p)= \begin{cases}\left(1,0,0,10^{-12}\right), & \text { if } x>\Delta x, y>\Delta y \\ \left(1,0,0, \frac{0.244816}{\Delta x \Delta y}\right), & \text { else. }\end{cases}
$$

The computational domain is $[0,1.1] \times[0,1.1]$. Reflective boundary conditions are applied along the bottom and left boundaries and the outflow conditions are imposed on the top and right boundaries. Figure 13A gives the density contours calculated by HM-WENO with $N_{x} \times N_{y}=160 \times 160$ at $t=1$, and the density profile along $y=0$ is shown in Figure 13B. The exact solution is specified in the work of Sedov. ${ }^{31}$ Numerical simulations show that all three schemes can solve this problem. It also can be found that, from the density distribution shown in Figure 13B, HM-WENO can still improve the solution near strong shock wave. 


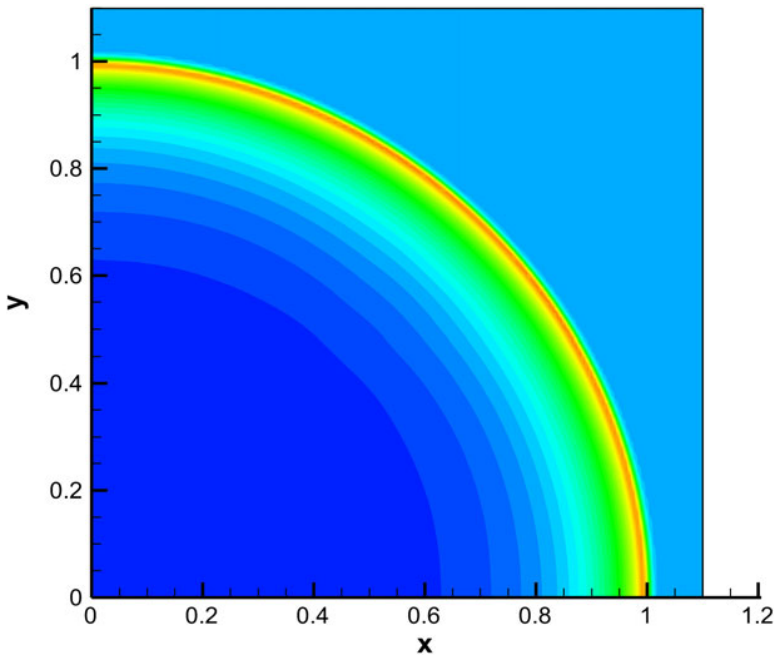

(A)

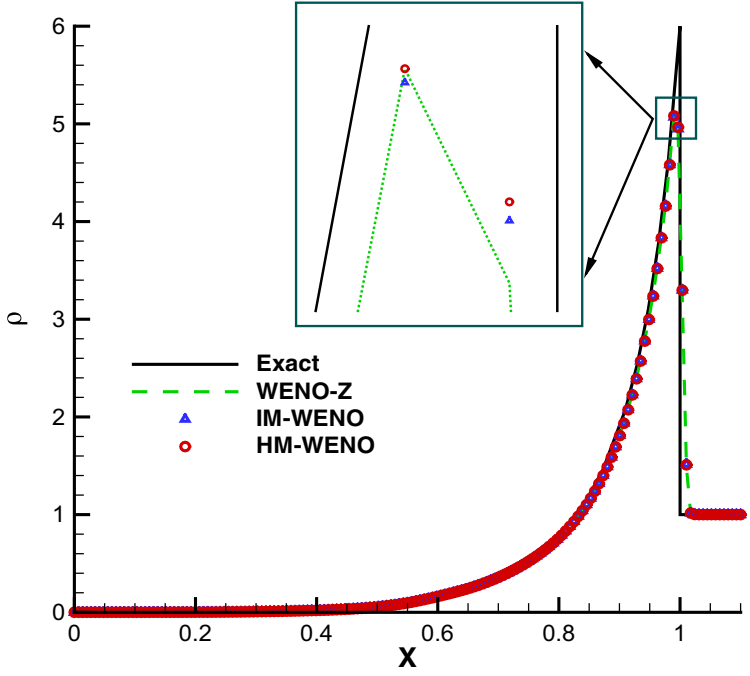

(B)

FIGURE 13 2D Sedov blast wave problem: A, Contour of density; B, Density distribution along $y=0$ [Colour figure can be viewed at wileyonlinelibrary.com]

\subsection{Shock wave impingement on a spatially evolving mixing layer}

This problem describes an oblique shock with angle $\beta=12^{\circ}$ impacts on a Mach number of 0.6 shear layer and is reflected by a wall at the lower boundary. ${ }^{33}$ The governing equation of this problem is $2 \mathrm{D}$ Navier-Stokes equations and is given by

$$
\frac{\partial U}{\partial t}+\frac{\partial F}{\partial x}+\frac{\partial G}{\partial y}=\frac{1}{R e}\left(\frac{\partial F_{v}}{\partial x}+\frac{\partial G_{v}}{\partial y}\right)
$$

where $U, F, G$, vectors are the same as given in Equation (68), and $F_{v}, G_{v}$ vectors are given by

$$
F_{v}=\left[\begin{array}{c}
0 \\
\tau_{x x} \\
\tau_{x y} \\
u \tau_{x x}+v \tau_{x y}+q_{x}
\end{array}\right], \quad G_{v}=\left[\begin{array}{c}
0 \\
\tau_{y x} \\
\tau_{y y} \\
u \tau_{y x}+v \tau_{y y}+q_{y}
\end{array}\right] \text {, }
$$

in which

$$
\begin{aligned}
& \tau_{x x}=\mu\left(\frac{4}{3} \frac{\partial u}{\partial x}-\frac{2}{3} \frac{\partial v}{\partial y}\right), \tau_{x y}=\tau_{y x}=\mu\left(\frac{\partial u}{\partial y}+\frac{\partial v}{\partial x}\right), \tau_{y y}=\mu\left(\frac{4}{3} \frac{\partial v}{\partial y}-\frac{2}{3} \frac{\partial u}{\partial x}\right), \\
& q_{x}=\mu \frac{1}{(\gamma-1) M^{2} P r} \frac{\partial T}{\partial x}, q_{y}=\mu \frac{1}{(\gamma-1) M^{2} P r} \frac{\partial T}{\partial y} .
\end{aligned}
$$

The computational domain is $[0,200] \times[-20,20]$. The inflow is specified as

$$
u=2.5+0.5 \tan h(2 y) .
$$

For the upper stream inflow $(y>0), \rho_{u}=1.6374, p_{u}=0.3327$; and for the lower stream inflow $(y<0), \rho_{l}=0.3626, p_{l}=$ 0.3327. The upper boundary is set as post-shock wave conditions, and a slip wall condition is applied at the lower wall boundary. Fluctuations are added to the vertical velocity component as

$$
v^{\prime}=\sum_{k=1}^{2} a_{k} \cos \left(2 \pi k t / T+\phi_{k}\right) \exp \left(-y^{2} / b\right),
$$

in which $b=10, a_{1}=a_{2}=0.05, \phi_{1}=0, \phi_{2}=\pi / 2, T=\lambda / u_{c}, \lambda=30, u_{c}=2.68$. The Prandtl number $\operatorname{Pr}$ is set to 0.72 , and the Reynolds number $R e$ is chosen to be 500. The Steger-Warming flux splitting method ${ }^{26}$ and WENO reconstruction for each component of split fluxes are also used for the inviscid convective fluxes, and the viscous terms are discretized with the fourth-order central difference scheme. Figure 14 gives the density contours with $N_{x} \times N_{y}=320 \times 80$ at $t=120$. An apparent difference is that, in the region of $x>120$, the present scheme obtains more clear vortex structures than the WENO-Z and IM-WENO schemes. 


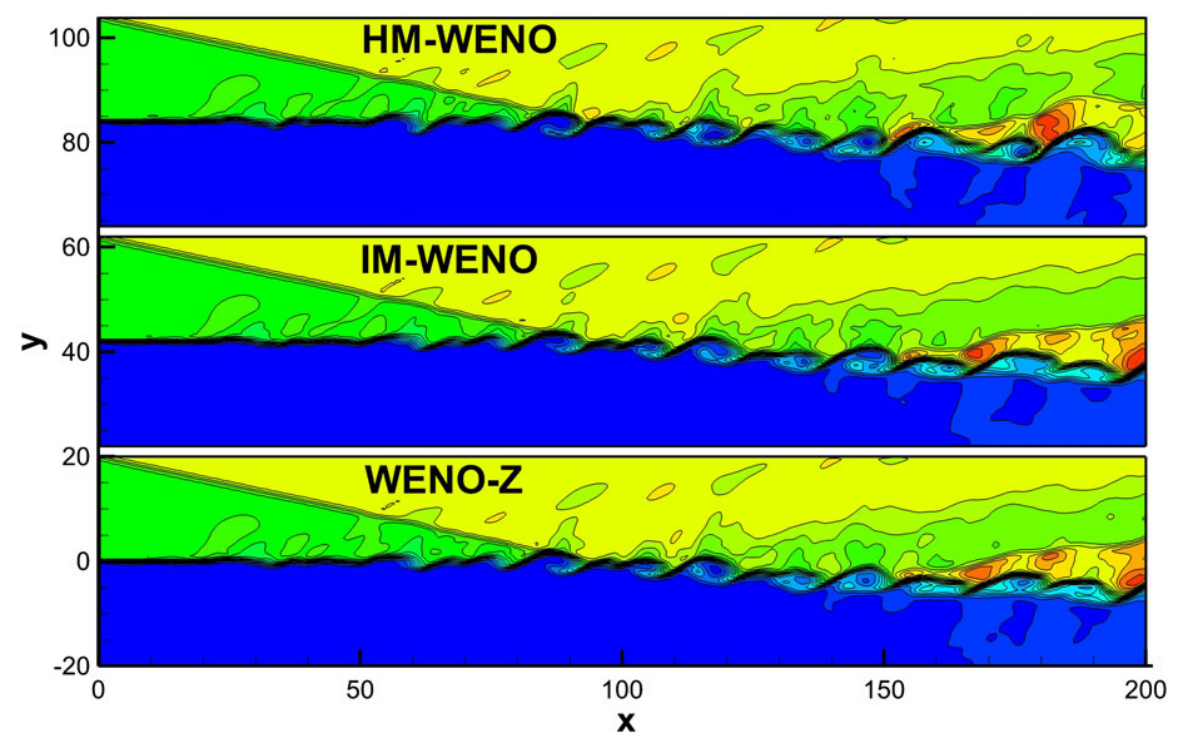

FIGURE 14 Shock/shear layer interaction problem, $N_{x} \times N_{y}=320 \times 80,20$ contours from 0.5 to 2.7 [Colour figure can be viewed at wileyonlinelibrary.com]

\section{5 | CONCLUSION REMARKS}

This paper analyzes the reason of several WENO schemes that failed to decrease the numerical dissipation near discontinuities. Then, a high performance fifth-order multistep WENO scheme is proposed by using a nonlinear function of two adjacent local smoothness indicators to replace previous linear combination. The function makes the calculated weights more accurate approximating to the ideal intermediate weights and, hence, decreases the numerical dissipation at transition points. The new scheme has the similar formula as those of the improved multistep WENO scheme suggested by Ma et $\mathrm{al}^{19}$; hence the resulted scheme is also more efficient than the original multistep WENO scheme of Shen et al. ${ }^{16}$ Meanwhile, theoretical analysis shows that the new scheme can improve the accuracy at transition points without reducing the accuracy in smooth regions including critical points. Various numerical examples are presented to demonstrate the high performance of the new scheme, such as the efficiency, the robustness, the high resolution of discontinuities, and the fifth-order accuracy in smooth regions.

\section{ACKNOWLEDGEMENTS}

This research work was supported by NSAF (No. U1530145), NKRDPC (No. 2016YFA0401200), SCP (No. TZ2016002), and NSFC (Nos. 11872067 and 91852203).

\section{ORCID}

Fangjun Zeng (iD) https://orcid.org/0000-0003-4192-7892

Shengping Liu (iD) https://orcid.org/0000-0001-9026-0436

\section{REFERENCES}

1. Liu XD, Osher S, Chan T. Weighted essentially non-oscillatory schemes. J Comput Phys. 1994;115:200-212.

2. Jiang G, Shu C. Efficient implementation of weighted ENO schemes. J Comput Phys. 1996;126:202-228.

3. Balsara D, Shu C. Monotonicity preserving weighted essentially non-oscillatory schemes with increasingly high order of accuracy. J Comput Phys. 2000;160:405-452.

4. Gerolymos G, Snchal D, Vallet I. Very-high-order WENO schemes. J Comput Phys. 2009;228(23):8481-8524. 
5. Henrick A, Aslam T, Powers J. Mapped weighted essentially non-oscillatory schemes: achieving optimal order near critical points. J Comput Phys. 2005;207:542-567.

6. Borges R, Carmona M, Costa B, Don W. An improved weighted essentially non-oscillatory scheme for hyperbolic conservation laws. J Comput Phys. 2008;227:3191-3211.

7. Castro M, Costa B, Don W. High order weighted essentially non-oscillatory WENO-Z schemes for hyperbolic conservation laws. J Comput Phys. 2011;230(5):1766-1792.

8. Ha Y, Kim C, Lee Y, Yoon J. An improved weighted essentially non-oscillatory scheme with a new smoothness indicator. J Comput Phys. 2013;232:68-86.

9. Fan P, Shen Y, Tian B, Yang C. A new smoothness indicator for improving the weighted essentially non-oscillatory scheme. $J$ Comput Phys. 2014;269:329-354.

10. Levy D, Puppo G, Russo G. Central WENO schemes for hyperbolic systems of conservation laws. ESAIM: M2AN. 1999;33(3):547-571.

11. Levy D, Puppo G, Russo G. A third order central WENO scheme for 2D conservation laws. Appl Numer Math. 2000;33(1):415-421.

12. Levy D, Puppo G, Russo G. A fourth-order central WENO scheme for multidimensional hyperbolic systems of conservation laws. SIAM J Sci Comput. 2002;24(2):480-506.

13. Martin M, Taylor E, Wu M, Weirs V. A bandwidth-optimized WENO scheme for the effective direct numerical simulation of compressible turbulence. J Comput Phys. 2006;220(1):270-289.

14. Balsara DS, Garain S, Shu CW. An efficient class of WENO schemes with adaptive order. J Comput Phys. 2016;326:780-804.

15. Shen YQ, Zha GC. Improvement of weighted essentially non-oscillatory schemes near discontinuities. Comput Fluids. 2014;96:1-9.

16. Shen YQ, Liu L, Yang Y. Multistep weighted essentially non-oscillatory scheme. Int J Numer Methods Fluids. 2014;75(4):231-249.

17. Peng J, Shen YQ. Improvement of weighted compact scheme with multi-step strategy for supersonic compressible flow. Comput Fluids. 2015;115:243-255.

18. Ghosh D, Baeder JD. Compact reconstruction schemes with weighted ENO limiting for hyperbolic conservation laws. SIAM J Sci Comput. 2012;34(3):A1678-A1706.

19. Ma YK, Yan ZG, Zhu HJ. Improvement of multistep WENO scheme and its extension to higher orders of accuracy. Int J Numer Methods Fluids. 2016;82(12):818-838.

20. van Lith BS, ten Thije Boonkkamp JH, IJzerman WL. Embedded WENO: a design strategy to improve existing WENO schemes. J Comput Phys. 2017;330:529-549.

21. Aràndiga F, Baeza A, Belda AM, Mulet P. Analysis of WENO schemes for full and global accuracy. SIAM J Numer Anal. 2011;49(2):893-915.

22. Don W-S, Borges R. Accuracy of the weighted essentially non-oscillatory conservative finite difference schemes. $J$ Comput Phys. 2013;250:347-372.

23. Cravero I, Semplice M. On the accuracy of WENO and CWENO reconstructions of third order on nonuniform meshes. J Sci Comput. 2016;67(3):1219-1246.

24. Shu CW, Osher S. Efficient implementation of essentially non-oscillatory shock-capturing schemes. J Comput Phys. 1988;77:439-471.

25. Shu C-W, Osher S. Efficient implementation of essentially non-oscillatory shock-capturing schemes, II. J Comput Phys. 1989;83(1):32-78.

26. Steger JL, Warming RF. Flux vector splitting of the inviscid gasdynamic equations with application to finite-difference methods. J Comput Phys. 1981;40(2):263-293.

27. Pirozzoli S. Conservative hybrid Compact-WENO schemes for shock-turbulence interaction. J Comput Phys. 2002;178(1):81-117.

28. Davoudzadeh F, McDonald H, Thompson BE. Accuracy evaluation of unsteady CFD numerical schemes by vortex preservation. Comput Fluids. 1995;24(8):883-895.

29. Balsara DS. Multidimensional HLLE Riemann solver: application to Euler and magnetohydrodynamic flows. $J$ Fluid Mech. 2010;229:1970-1993.

30. Woodward P, Colella P. The numerical simulation of two-dimensional fluid flow with strong shocks. J Comput Phys. 1984;54(1):115-173.

31. Sedov LI. Similarity and Dimensional Methods in Mechanics. New York, NY: Academic Press; 1959.

32. Zhang XX, Shu CW. Positivity-preserving high order finite difference WENO schemes for compressible Euler equations. J Comput Phys. 2012;231(5):2245-2258.

33. Yee HC, Sandham ND, Djomehri MJ. Low-dissipative high-order shock-capturing methods using characteristic-based filters. $J$ Comput Phys. 1999;150(1):199-238.

How to cite this article: Zeng F, Shen Y, Liu S, Liu L. A high performance fifth-order multistep WENO scheme. Int J Numer Meth Fluids. 2019;91:159-182. https://doi.org/10.1002/fld.4747 


\section{APPENDIX}

The process for analyzing the accuracy of the IM-WENO and HM-WENO schemes in smooth regions including critical points is presented here, where the symbols $p$ and $m$ represent + and - , respectively.

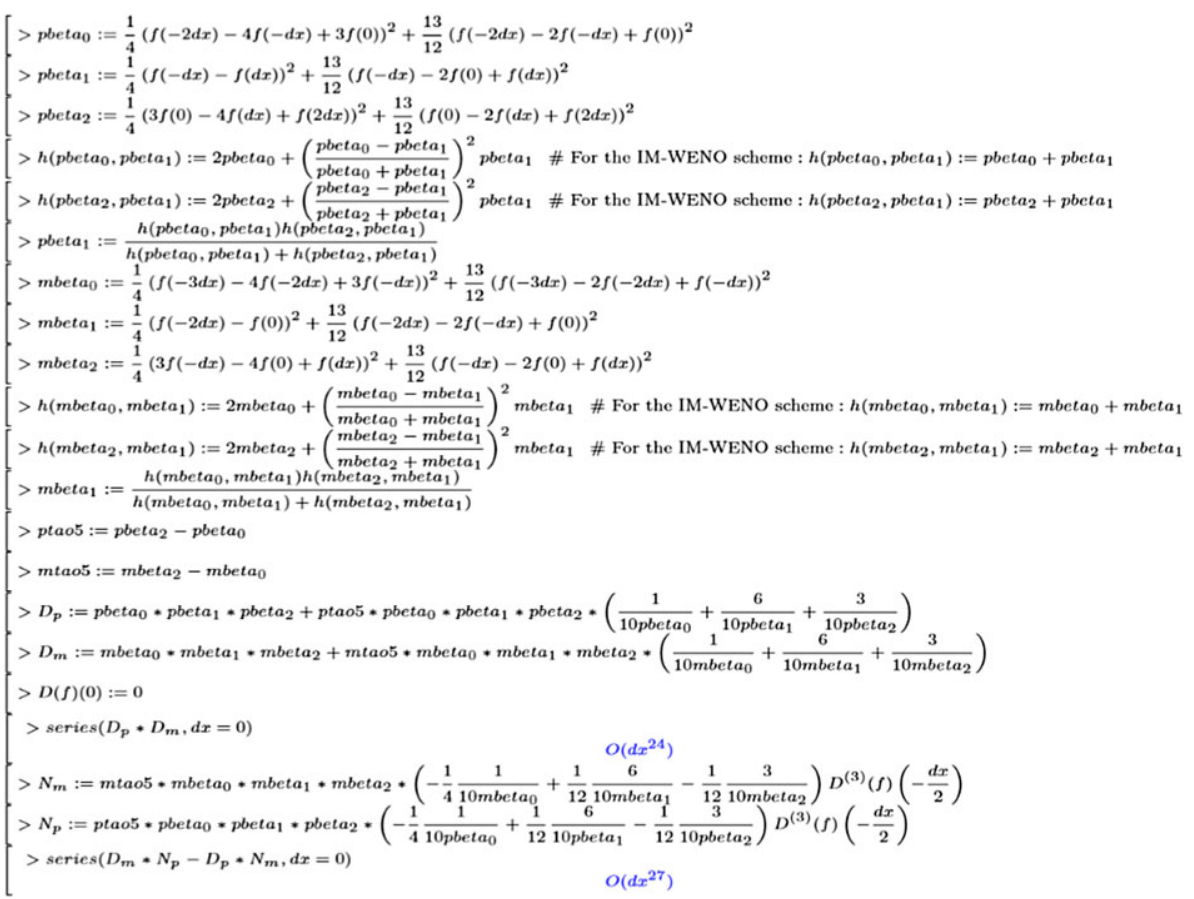

